OPEN ACCESS

Edited by:

Hua Zhu,

The Ohio State University, United States

Reviewed by:

Tahir Hussain,

University of Houston, United States John D Imig,

Medical College of Wisconsin, United States

*Correspondence: Lakshmi Pulakat

lpulakat@tuftsmedicalcenter.org

tThese authors have contributed equally to this work

Specialty section: This article was submitted to Integrative and Regenerative Pharmacology,

a section of the journal

Frontiers in Pharmacology

Received: 09 April 2021 Accepted: 31 May 2021

Published: 18 June 2021

Citation:

Gavini MP, Mahmood A,

Belenchia AM, Beauparlant $P$, Kumar SA, Ardhanari S, DeMarco VG and Pulakat $L$ (2021) Suppression of Inflammatory Cardiac Cytokine Network in Rats with Untreated Obesity and Pre-Diabetes by AT2

Receptor Agonist NP-6A4.

Front. Pharmacol. 12:693167. doi: 10.3389/fphar.2021.693167

\section{Suppression of Inflammatory Cardiac Cytokine Network in Rats with Untreated Obesity and Pre-Diabetes by AT2 Receptor Agonist NP-6A4}

Madhavi P. Gavini ${ }^{1 \dagger}$, Abuzar Mahmood ${ }^{2,3,4 \dagger}$, Anthony M. Belenchia 2,5 , Paige Beauparlant ${ }^{2,3,5}$, Senthil A. Kumar ${ }^{3}$, Sivakumar Ardhanari ${ }^{3}$, Vincent G. DeMarco ${ }^{2,3,4}$ and Lakshmi Pulakat ${ }^{2,3,4,5,6 *}$

${ }^{1}$ Novopyxis Inc., Boston, MA, United States, ${ }^{2}$ Dalton Cardiovascular Research Center, Columbia, MO, United States, ${ }^{3}$ Department of Medicine, Boston, MA, United States, ${ }^{4}$ Harry S. Truman Memorial VA Hospital, Columbia, MO, United States, ${ }^{5}$ Department of Nutrition and Exercise Physiology, University of Missouri, Columbia, MO, United States, ${ }^{6}$ Tufts Medical Center and Department of Medicine, Molecular Cardiology Research Institute, Tufts University School of Medicine, Boston, MA, United States

Obesity affects over $42 \%$ of the United States population and exacerbates heart disease, the leading cause of death in men and women. Obesity also increases pro-inflammatory cytokines that cause chronic tissue damage to vital organs. The standard-of-care does not sufficiently attenuate these inflammatory sequelae. Angiotensin II receptor AT2R is an antiinflammatory and cardiovascular protective molecule; however, AT2R agonists are not used in the clinic to treat heart disease. NP-6A4 is a new AT2R peptide agonist with an FDA orphan drug designation for pediatric cardiomyopathy. NP-6A4 increases AT2R expression (mRNA and protein) and nitric oxide generation in human cardiovascular cells. AT2R-antagonist PD123319 and AT2RSiRNA suppress NP-6A4-effects indicating that NP-6A4 acts through AT2R. To determine whether NP-6A4 would mitigate cardiac damage from chronic inflammation induced by untreated obesity, we investigated the effects of 2-weeks NP-6A4 treatment $(1.8 \mathrm{mg} / \mathrm{kg}$ delivered subcutaneously) on cardiac pathology of male Zucker obese (ZO) rats that display obesity, pre-diabetes and cardiac dysfunction. NP-6A4 attenuated cardiac diastolic and systolic dysfunction, cardiac fibrosis and cardiomyocyte hypertrophy, but increased myocardial capillary density. NP-6A4 treatment suppressed tubulointerstitial injury marker urinary $\beta-N A G$, and liver injury marker alkaline phosphatase in serum. These protective effects of NP-6A4 occurred in the presence of obesity, hyperinsulinemia, hyperglycemia, and hyperlipidemia, and without modulating blood pressure. NP-6A4 increased expression of AT2R (consistent with human cells) and cardioprotective erythropoietin (EPO) and Notch1 in ZO rat heart, but suppressed nineteen inflammatory cytokines. Cardiac miRNA profiling and in silico analysis showed that NP$6 \mathrm{~A} 4$ activated a unique miRNA network that may regulate expression of AT2R, EPO, Notch1 and inflammatory cytokines, and mitigate cardiac pathology. Seventeen proinflammatory and pro-fibrotic cytokines that increase during lethal cytokine storms caused by infections such as COVID-19 were among the cytokines suppressed by NP-6A4 
treatment in $\mathrm{ZO}$ rat heart. Thus, NP-6A4 activates a novel anti-inflammatory network comprised of 21 proteins in the heart that was not reported previously. Since NP-6A4's unique mode of action suppresses pro-inflammatory cytokine network and attenuates myocardial damage, it can be an ideal adjuvant drug with other anti-glycemic, antihypertensive, standard-of-care drugs to protect the heart tissues from proinflammatory and pro-fibrotic cytokine attack induced by obesity.

Keywords: obesity, cardiac dysfunction, inflammatory cytokines, myocardial strain, AT2 receptor, heart disease, NP-6A4

\section{INTRODUCTION}

Heart disease, the number one killer of men and women, is induced by obesity that affects $42.4 \%$ of the United States population (WHO 2020; Virani et al., 2021; Piché et al., 2020). Obesity induces pre-diabetes and cardiometabolic disease via increasing the levels of pro-inflammatory and pro-fibrotic cytokines that cause cardiac fibrosis, hypertrophy, reduction in capillary density (capillary rarefaction) and significant myocardial damage (Cavalera et al., 2014; Riaz et al., 2018; Strain and Paldánius, 2018; CDC: Centers for Disease Control and Prevention, 2020; CDC: Centers for Disease Control and Prevention, 2021). Obesity also increases the severity and lethality of infections by exacerbating the underlying cardiac damage (Mauvais-Jarvis., 2020; Tartof et al., 2020). Conventional cardio-protective and anti-inflammatory drugs are insufficient to attenuate this inflammatory onslaught and subsequent myocardial structural damage in obese patients. Thus, there is a critical need for new treatment paradigms to protect the heart from obesity-induced cardiometabolic disease (Fang et al., 2017;
Levick and Widiapradja, 2020). Angiotensin II (Ang II) receptor (AT2R) is an anti-inflammatory molecule and a promising target for therapeutic intervention (Chow et al., 2016; Kaschina et al., 2017; Bennion et al., 2018). Transgenic overexpression of AT2R in murine models is cardioprotective, and its deficiency is implicated in myocardial damage in obese and diabetic rats and humans (Altarche-Xifró et al., 2009; Tousoulis et al., 2010; Qi et al., 2012; Chow et al., 2016; Lum-Naihe et al., 2017). There are no AT2R agonists in clinic for heart disease. We and others have shown that AT2R activates reparative signaling and attenuates inflammatory signaling by the AT1R subtype that induces hypertension, fibrosis and cardiac structural damage (Kumar et al., 2002; Kurisu et al., 2003). Therefore, we hypothesized that increasing the expression and antiinflammatory signaling by AT2R would mitigate pathologic cardiac remodeling and cardiac dysfunction caused by obesityinduced increases in pro-inflammatory and pro-fibrotic cytokines.

NP-6A4 is a $768 \mathrm{Da}$, six amino acid peptide drug (Figure 1A) designed to specifically bind and activate AT2R and has an FDA
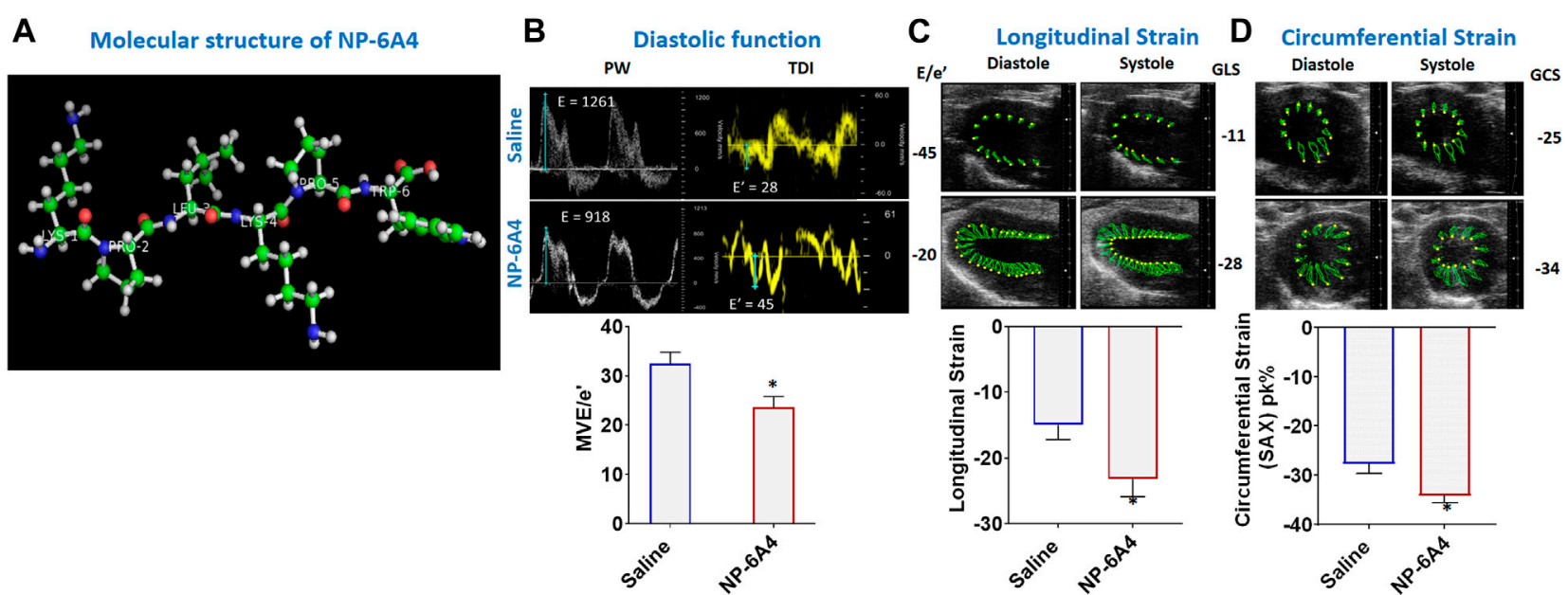

FIGURE 1 | NP-6A4 attenuates cardiac diastolic dysfunction and improves myocardial longitudinal and circumferential strain in ZO rats with untreated diabetes and obesity. (A) Structure of NP-6A4, a patented six amino acid peptide agonist of the AT2R with the orphan drug designation for pediatric cardiomyopathy from the FDA. (B) Representative Pulse Wave Doppler and Tissue Doppler spectra from a saline- (top panels) or NP-64A-treated (bottom panels) ZO rat. Note the lower E/e' ratio in the NP-64A4 treated rat compared to the untreated rat, indicating lower LV filling pressure with NP-64A treatment. (C) Representative images taken in the parasternal long axis view from a saline-treated rat (top panes) taken at end-diastole and end-systole indicate endocardial longitudinal deformation during the cardiac cycle compared to those from an NP-64A4-treated rat (bottom panels). (D) Representative images taken in the short axis view from a saline-treated rat (top panels) taken at end diastole and end systole indicate endocardial circumferential deformation during the cardiac cycle compared to those from an NP-64A4-treated rat (bottom panels). 
designation for pediatric cardiomyopathy (FDA US Food and Drug Administration 2017). We showed that NP-6A4 treatment increased survival of mouse cardiomyocyte HL-1 cells, and human coronary artery vascular smooth muscle cells (hCAVSMCs) subjected to acute nutrient stress better than four $\beta 1$-adrenergic receptor blockers (atenolol, metoprolol, carvedilol and nebivolol) and the AT1R blocker losartan (Mahmood and Pulakat, 2015). We also reported that NP-6A4 increases 1) AT2R expression and signaling in hCAVSMCs and endothelial cells (ECs), 2) cellular respiration of hCAVSMCs, 3) nitric oxide generation by ECs, and 4) reduces reactive oxygen species induced by exposure to Doxorubicin in hCAVSMCs (Toedebusch et al., 2018). The protective effects of NP-6A4 on hCAVSMCs and hCAECs were inhibited by pretreatment with the AT2R-specific antagonist PD123319 or antiAT2R siRNA, confirming NP-6A4-effects are mediated through AT2R (Mahmood and Pulakat, 2015; Toedebusch et al., 2018). Moreover, NP-6A4 reduced aortic stiffness and proteolytic activity in a mouse model (Sharma et al., 2020). Since NP-6A4 could increase AT2R expression and signaling, we tested whether NP-6A4 could mitigate pathologic cardiac remodeling and dysfunction caused by a combination of obesity and T2DM in a rat model.

We chose male Zucker obese (ZO) rats (Strain Code 185 from Charles River) that harbor a leptin receptor mutation (fa/fa) that causes hyperphagia and exhibit reduction in capillary density (capillary rarefaction), and cardiac fibrosis as the rat model for this study (Zhou et al., 2010; Frisbee et al., 2014; Luck et al., 2017). By the age of nine weeks, $\mathrm{ZO}$ rats exhibit severe obesity and insulin resistance, cardiac dysfunction with preserved ejection fraction, a characteristic cardiac pathology of obese and prediabetic individuals (Zhou et al., 2010; Maack et al., 2018). Therefore, male $\mathrm{ZO}$ rat is an appropriate pre-clinical model for evaluating the cardioprotective effects of NP-6A4 in conditions of untreated obesity. Supplementary Table S1 confirms these metabolic features of 10 -week old male $\mathrm{ZO}$ rats compared to non-diabetic lean rats. Since our goal was to test whether NP-6A4 could mitigate cardiac structural and functional damage in the presence of untreated obesity and pre-diabetes, we did not treat ZO rats used in this study with any of the established anti-glycemic, anti-hypertensive or anti-lipidemic drugs. At the age of 11-weeks ZO rats were treated with either saline or NP-6A4 $(1.8 \mathrm{mg} / \mathrm{kg} / \mathrm{day})$ for a two-week period. To gain mechanistic insight into NP-6A4's mode of action, we investigated how it modulated cardiac cytokines. Here we report that NP-6A4 attenuated cardiac functional and structural damage in obese and pre-diabetic $\mathrm{ZO}$ rats and suppressed a large array of inflammatory cytokines in the heart that are implicated in cytokine storm caused by chronic and acute cardiac inflammation caused by metabolic and infectious diseases.

\section{MATERIALS AND METHODS}

\section{Animals, Fasting Plasma Profile, HbA1c, and Urine Chemistry}

All animal procedures used in this study were approved prior to the beginning of these studies by the Harry S. Truman Memorial
Veterans Hospital (HSTMVH) Subcommittee for Animal Safety and University of Missouri IACUC. All animals were cared for in accordance with the Guidelines for the Care and Use of Laboratory Animals (National Institutes of Health publication 85-23). Male ZO rats from Charles River Laboratories were used for these studies ( $n=26$ rats). All animals were given numbers after randomization based on body weight and before starting treatments and only the numbers were used for identifying the animals during procedures to ensure studies/analyses were performed in a blinded manner. Male Zucker lean (ZL) rats $(n=7)$ were used to compare metabolic parameters. 11-weeks of age, and presence of obesity and pre-diabetes as determined by body weight and fasting glucose levels before the beginning of treatments were the inclusion criteria. Only male $\mathrm{ZO}$ rats were used in this study since female $\mathrm{ZO}$ rats at this age and fed with normal chow do not develop hyperglycemia, and the goal of the study was to test the effect of NP-6A4 on obesity- and prediabetes-induced heart disease with preserved ejection fraction. To evaluate the effect of a 2-weeks treatment with NP-6A4 on cardiac structure and function, 11-week old $\mathrm{ZO}$ rats were used. $\mathrm{NP}-6 \mathrm{~A} 4$ dissolved in saline $(1.8 \mathrm{mg} / \mathrm{kg} /$ day; $500 \mu \mathrm{l}$ volume $)$ was delivered subcutaneously via daily injection and saline was used as control. Rats were maintained on ad libitum food and water and housed singly at the HSTMVH animal housing facility under standard laboratory conditions. Room temperature was maintained at $21-22^{\circ} \mathrm{C}$. Light and dark cycles were for $12 \mathrm{~h}$, but animals were entrained to have dark cycle (awake time) during the day and light cycle (sleep time) during the night to prevent loss of their sleep time during the experiments. Fasting plasma profile was determined by collecting blood from the saphenous vein as described previously (Luck et al., 2017; Lum-Naihe et al., 2017). Percentage of HbA1c in blood was measured just before euthanasia in animals fasted for $6 \mathrm{~h}$ using a DCA Vantage Analyzer (Siemens, Malvern, PA). Urine was collected by placing rats in metabolic chambers for $24 \mathrm{~h}$ according to the protocol approved by the University of Missouri-Columbia IACUC and stored frozen at $-80^{\circ} \mathrm{C}$ until further use. For glucose, enzymatic creatinine (Diazyme, Poway,California,United States), $\mathrm{N}$-acetyl- $\beta$ - glucosamynidase $(\beta-\mathrm{NAG})$, urine protein (Beckman Coulter), and Gamma Glutamyl Transferase (GGT) measurements, commercially available assays were used on the AU680 automated clinical chemistry analyzer as reported previously (Nistala et al., 2017). Electrolytes ( $\mathrm{Na}$ and $\mathrm{K}$ ) were measured using ion-specific electrodes on the AU680 as well as we have done before (Nistala et al., 2017). Tissues were harvested at the time of euthanasia as described before (Luck et al., 2017), weighed, flash frozen in liquid nitrogen, and stored at $-80^{\circ} \mathrm{C}$ for future use. Wet weights of heart, liver, lung and kidney were determined at the time of euthanasia.

\section{Echocardiography}

Transthoracic echocardiography on saline- or NP-6A4-treated $\mathrm{ZO}$ rats was performed under inhaled isoflurane anesthesia (1.5-2.0\% maintenance) utilizing a Vevo2100 dedicated rodent ultrasound imaging system (FUJIFILMS, Visualsonics, Toronto) with an MS250S high frequency echo probe at the Small Animal 
Ultrasound Imaging Center at the Harry S Truman VA Research Center as described previously (Lum-Naihe et al., 2017). Speckletracking based strain analysis of B-Mode ultrasound images was performed in the parasternal long- and short-axis views (PLAX and SAX, respectively), as described previously (Lum-Naihe et al., 2017). Images were acquired at the highest frame rates possible (>200 frames per second). PLAX views were used for evaluation of longitudinal strain and strain rate. SAX views that were acquired at the mid-papillary level, were used for evaluation of circumferential and radial strain analyses. Strain analyses were conducted by following the procedure described previously (Lum-Naihe et al., 2017) by using the manufacturer supplied speckle-tracking algorithm (VevoStrain ${ }^{\circledR}$, VisualSonics). Briefly, at least three of the highest quality B-mode loops were chosen, i.e., those with little gel artifact or obstruction from ribs, as well as those that display the endocardial and epicardial borders throughout the cine loop for each animal. Initially, the endocardial and epicardial borders were traced with the cine loop stopped at end diastole. Cine-loops were replayed to confirm good border tracking over all cardiac cycles and tracking adjustments were made as needed. The final tracked images were then evaluated for strain measurements. Strain measures were averaged over the cardiac cycles yielding curvilinear strain and strain rate data. Global strain values, peak strain and strain rate measurements of NP-6A4 treated rats were compared to those of saline-treated rats. These procedures for data analysis were repeated independently by two qualified individuals for additional confirmation. Blood pressure was measured noninvasively on conscious rats using a CODA volume pressure recording tail-cuff system (Kent Scientific Corporation, Torrington, CT) as described previously (Sharma et al., 2020). All analysis was done using animal numbers to ensure blinded data analysis.

\section{Histopathology}

Tissues from animals (randomly numbered) were fixed in $10 \%$ neutral buffered formalin (NBF), embedded into paraffin blocks, sections were cut at $4 \mu \mathrm{m}$ thickness and used for histopathology as described previously (Luck et al., 2017). To determine interstitial fibrosis, heart sections were stained with Picrosirius Red at Research Animal Diagnostic Laboratory (RADIL), Columbia, MO. The stained sections were scanned using the Aperio CS Slide Scanner by WSI Analytics Lab, Department of Pathology and Anatomical Sciences, University of Missouri, Columbia, MO. Scanned sections were visualized using Aperio ImageScope (Leica Biosystems). Next, 10 interstitial ( $\times 20$ magnification) images of the most fibrotic regions were selected per animal. Fibrotic area was quantified using the in-built Positive Pixel Count (V9) algorithm (settings were manually determined as follows: hue value $=0$; hue width $=0.154$; color saturation threshold $=0.04$ ) Positivity (Positive/Total Pixels) was averaged over all regions from a single group to determine mean fibrotic area per group.

To determine cardiomyocyte size and capillary density, heart sections were dewaxed in CitriSolv (Fisher Scientific), rehydrated in an ethanol series and HEPES wash buffer, followed by a heatmediated antigen retrieval step in sodium citrate buffer. To block non-specific binding sites, sections were incubated with blocking buffer ( $10 \%$ donkey serum, $1 \%$ BSA) for $2 \mathrm{~h}$ at room temperature, followed by incubation with Helix pomatia agglutinin (HPA) conjugated to Alexa Fluor 647 (Life Technologies; 1:400, $2.5 \mu \mathrm{g} / \mathrm{ml}$ ) and Griffonia simplicifolia isolectin B4 (IB4) conjugated to Alexa Fluor 594 (Life Technologies; 1:200, $5 \mu \mathrm{g}$ / $\mathrm{ml}$ ) for $4 \mathrm{~h}$ at room temperature. Sections were thoroughly washed and slides were mounted using Fluoroshield with DAPI (Sigma). Imaging was performed using a Leica DMI4000B inverted confocal microscope at 40X and 63X. Cell size and capillary density analysis was done using code written inhouse using the EBImage package in R (Pau et al., 2010). Briefly, for measuring cardiomyocyte cell size, HPA stained images were thresholded by intensity to produce binary masks. The masks were used to select closed contours as potential cells. Objects of interest were filtered by morphological parameters (such as area, perimeter, and eccentricity), and subsets of the remaining cells were randomly selected for inclusion in analysis. Following the initial segmentation, artifacts (such as merged cells, split cells, or recognition of non-cell objects) were identified and removed by a user. Area was then computed for approved cells. Cell sizes of a minimum of 65 cells per animal were determined and a minimum of five animals were used per group. A similar protocol was used to extract capillaries for IB4 stained images. Following initial extraction, errors in capillary recognition were manually corrected by a user. All capillaries present in an image were used to calculate the final density. Data are presented as mean \pm SEM.

\section{Immunohistochemistry}

Five micrometer heart sections from the saline and NP-6A4 treated $\mathrm{ZO}$ rats were deparaffinized in xylene (Fisher Scientific), rehydrated in an ethanol series and HEPES wash buffer, followed by a heat-mediated antigen retrieval step in sodium citrate buffer. Following rehydration, sections were incubated with Wheat germ agglutinin (WGA) conjugated to Alexa Fluor 647 (Life Technologies; 1:200, $5.0 \mu \mathrm{g} / \mathrm{ml}$ ). After washing, tissue sections were permeabilized with $0.2 \%$ Triton-X (ThermoFisher) in PBS and subsequently blocked as described above independently for each AT2R, ACE2, and MAS staining. Antibodies for AT2R (Ab19134), ACE2 (Ab87436), and MAS (Ab66030) were from Abcam Biotech. Sections were thoroughly washed and slides were mounted using Fluoroshield with the nuclear counterstain, DAPI (Sigma). Imaging was performed using a Leica DMI4000B inverted confocal microscope at $20 \mathrm{X}$ and 63X. Fluorescence intensity (relative to nuclei) was subsequently quantified using ImageJ software (NIH, Bethesda, MD) using a minimum of 10 regions of interest per section.

\section{Intracardiac Cytokine Analysis Using Quantibody ${ }^{\circledR}$ Rat Cytokine Array 67 and IPA Analysis}

Heart tissues that were frozen at $-80^{\circ} \mathrm{C}$ were homogenized and lysates were prepared for Raybiotech's Quantibody ${ }^{\circledR}$ Rat Cytokine Array 67 analysis as described previously (LumNaihe et al., 2017). Cytokine analysis of the heart tissue lysates was performed by Raybiotech according to their protocol and 
software analysis. Analysis was performed for 67 nonoverlapping cytokines in quadruplicates per animal along with appropriate positive and negative controls. Cytokines that exhibited statistically significant differences $(p<0.05$, Paired Student's $t$-Test) between different groups and at least a 1.5fold change in either direction (increased or decreased) were selected for input into Ingenuity Pathway Analysis (IPA, Qiagen, Germantown, MD) to identify diseases and functions that were affected. Heatmaps were generated using the ggplot2 package for R (Wickham 2016).

\section{Intracardiac miRNA Expression Analysis}

Total RNA was isolated from the frozen $\left(-80^{\circ} \mathrm{C}\right)$ heart tissues of saline- or NP-6A4 treated rats using the mirVana ${ }^{\mathrm{TM}}$ miRNA isolation kit and GeneChipTM miRNA 4.0 array was used for determining the expression of cardiac miRNA in these isolated total RNA samples as described previously (Belenchia et al., 2018). Briefly, Affymetrix ${ }^{\circledR}$ FlashTag $^{\mathrm{TM}}$ Biotin HSR RNA Labeling Kit specifically designed for GeneChipTM miRNA array (ThermoFisher Scientific catalog number 90911) was used for preparing the targets ( $n=5$ animals per group). Hybridization of the target to the GeneChipTM miRNA 4.0 array for $16 \mathrm{~h}$ followed by scanning of the fluorescence intensity emitted by the labeled cRNA/cDNA bound to the probe arrays using the Affymetrix GeneChip scanner 3,000 $7 \mathrm{Gw}$ was performed at the University of Colorado Microarray Core Lab for a fee. Data were collected using Affymetrix Command Console Software at the University of Colorado Microarray Core Lab. Affymetrix Expression Console TM software was used to process the CEL files and normalize data. A two-sample $t$-test was used to compare the two groups for each miRNA and identify the differentially expressed miRNAs that showed statistical significance $(p<0.05)$. The heatmap was generated using the ggplot2 package for R (Wickham 2016).

\section{RNA Isolation and Quantitative Real Time-PCR}

Cardiac expression of Agtr2 (AT2R), Agtr1a (AT1A), Agtr1b (AT1B), miR-7a-1-3p, miR-138-5p, miR-148b-3p, and miR101b-3p miRNAs were determined using mRNA and miRNA isolated from frozen $\mathrm{ZO}$ rat heart tissues ( $n=5$ per group) as described previously (Luck et al., 2017; Lum-Naihe et al., 2017; Belenchia et al., 2018). Agtr2, At1ra, At1rb, and 18s PCR reactions were performed in triplicate using TaqMan Fast Universal PCR Master Mix (2X) and TaqMan Assays with primers specific to the genes of interest (Applied Biosystems). miRNA real-time PCR reactions were performed in triplicate using either TaqMan Fast Universal PCR Master Mix (2X) (Applied Biosystems) or miScript II Mix (Qiagen). TaqMan Advanced MicroRNA Assays (Life Technologies) primers were used for miR-7a-1-3p and miR-138-5p. miScript II Assay Primers (Qiagen) were used for miR-148-3p, miR-101b-3p, and miR-190a-3p.

\section{Statistical Analysis}

All data were assessed for homoscedasticity and normality by Shapiro-Wilk test. For the comparison of the two groups, unpaired two-tailed t-tests were performed, with Mann-Whitney correction used in cases of non-parametric data. A $p$-value $<0.05$ was deemed significant.

\section{RESULTS}

\section{NP-6A4 Treatment Attenuated Cardiac Diastolic Dysfunction in ZO Rats Without Changing Their Blood Pressure or Other Metabolic Parameters}

Mitral E/e' ratio is a noninvasive measure of left ventricular filling pressure. Elevated $\mathrm{E} / \mathrm{e}^{\prime}$ ratio is considered as a cardinal sign for heart failure with preserved ejection fraction (HFpEF) (Sharp et al., 2010; Zoppini et al., 2018). In diabetic humans, E/e' ratio is significantly increased compared to non-diabetic controls indicating diastolic dysfunction (Zoppini et al., 2018). A unit rise in the $\mathrm{E} / \mathrm{e}^{\prime}$ ratio was associated with a $17 \%$ increment in risk of a cardiac events as shown in a study of 980 participants in the Anglo-Scandinavian Cardiac Outcomes Trial (ASCOT) (Sharp et al., 2010). In healthy rats, E/e' is in the range of 18-20. In 13week old obese and pre-diabetic $\mathrm{ZO}$ rats treated with saline, average E/e' was-31.94 \pm 3.24 . However, in $\mathrm{ZO}$ rats treated with NP-6A4, E/e' was lowered by an average of 8.5 points and a maximum of 25 points compared to saline-treated $\mathrm{ZO}$ rats (Figure 1B; Supplementary Table S2). This observation indicated that NP-6A4 treatment mitigated diastolic dysfunction in $\mathrm{ZO}$ rats with untreated obesity and T2DM. The myocardial performance index (MPI) is a Doppler-derived index of global LV function and a negative prognostic marker for congestive heart failure when elevated (Arnlov et al., 2004). NP-6A4 treatment significantly lowered MPI also in ZO rats (Supplementary Table S2) further confirming that NP-6A4 is cardio-protective.

NP-6A4 treatment did not change body weight, fasting glucose, insulin, and triglycerides during the course of treatment (Figures 2A-D). Analysis of plasma at the time of euthanasia showed that no significant changes occurred in the levels of cholesterol, high density and low density lipoprotein (HDL and LDL), Hemoglobin Alc Alanine Aminotransferase, Asparatate Aminotransferase and uric acid in response to NP6A4 treatment (Figures 2E-I). Previous reports show that central infusion with AT2R agonists could reduce blood pressure, however, systemic treatment did not change blood pressure (Sumners et al., 2015; Dai et al., 2016) in different murine models. To verify whether the improvement in cardiac functions of $\mathrm{ZO}$ rats resulted from any changes in blood pressure, we tested the effect of four-week NP-6A4 treatment on blood pressure in a separate cohort of $\mathrm{ZO}$ rats. Diastolic, systolic and mean arterial pressure were not significantly different between saline- and NP-6A4 treated ZO rats (Supplementary Table S2). This is consistent with other reports that show systemic administration of AT2R agonists are not effective in changing blood pressure. Moreover, this is also consistent with NP-6A4's effect on blood pressure in a mouse model for aneurism (Sharma et al., 2020). Thus, the NP-6A4-mediated effects on cardiac functions are independent of blood pressure. 

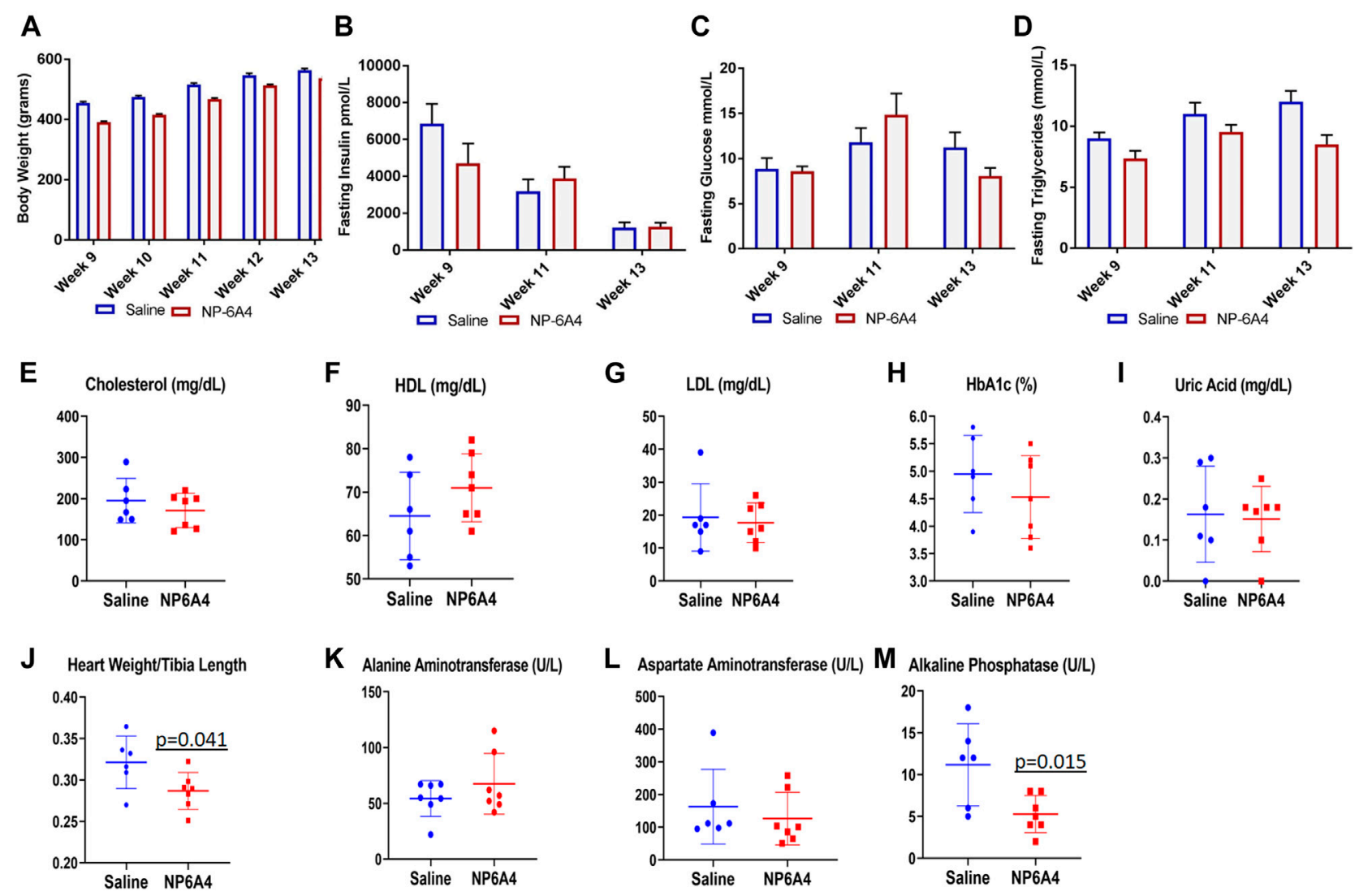

FIGURE 2 | NP-6A4 treatment did not change levels of fasting plasma glucose, insulin, lipids, and aminotransferases, but reduced heart weight and alkaline phosphatase significantly. $\mathrm{N}=6$ for saline group and seven for NP-6A4 group. Heart weight (grams)/tibia length (cm) and plasma levels of alkaline phosphatase (U/L) at the end of treatment were significantly reduced ( $p$ values are marked). None of the other parameters showed any significant changes.

\section{NP-6A4 Treatment Improved Myocardial Global Circumferential Strain and Longitudinal Strain, Reduced Cardiac Fibrosis, Heart Weight and Cardiomyocyte Hypertrophy in ZO Rats}

In patients having heart disease with preserved ejection fraction, impairment of left ventricular global longitudinal strain (GLS) is associated with a 5.6-fold increase in mortality (Blomstrand et al., 2015; Krishnasamy et al., 2015). Moreover, impairment of circumferential strain is a negative prognosticator for heart failure in asymptomatic subjects without previous clinical CVD (Choi et al., 2013). Speckle tracking echocardiography analysis (Lum-Naihe et al., 2017) revealed that myocardial longitudinal strain and strain rate, and circumferential strain and strain rate were significantly improved in NP-6A4 treated rats compared to saline treated rats (Figures 1C,D and Supplementary Table S2).

The $\mathrm{ZO}$ rats are reported to exhibit cardiac fibrosis starting at the age of 9 weeks (Zhou et al., 2010). The ZO rats used in this study were 13-weeks old at the end of NP-6A4 treatment. Therefore, we tested whether NP-6A4 treatment modulated their cardiac fibrosis. Picrosirius red (PSR) staining (Vogel et al., 2015) was used to visualize collagen content in heart tissue sections from saline- and NP-6A4 treated $\mathrm{ZO}$ rats. There was significant reduction in PSR staining in NP-6A4-treated ZO rat hearts (Figures 3A,B). This suggests that NP-6A4 reduces excessive collagen and thereby attenuates cardiac fibrosis. The wet heart weight adjusted to tibia length was significantly reduced in response to NP-6A4 treatment in $\mathrm{ZO}$ rats (Figure 2J) indicating suppression of cardiac hypertrophy. Cardiomyocyte hypertrophy is often seen in obese and diabetic patients (Oktay et al., 2000) and reported in ZO rats (Martinelli et al., 2020). To test if NP-6A4 modulated cardiomyocyte hypertrophy in obese and pre-diabetic $\mathrm{ZO}$ rats, we performed immunofluorescent cell membrane staining using Helix pomatia agglutinin (HPA) conjugated to Alexa Fluor 647 to evaluate cardiomyocyte size (Lum-Naihe et al., 2017). As shown in Figures 3C,D, NP-6A4 treated hearts had a significant reduction in cardiomyocyte size indicating that the short-term NP-6A4 treatment could reduce cardiomyocyte hypertrophy.

\section{NP-6A4 Treatment Increased Cardiac Capillary Density in ZO Rats}

In patients with chronic heart failure clinical research has shown that there is a $\sim 20 \%$ reduction in their skeletal muscle capillary density and that was inversely related to their maximum oxygen 

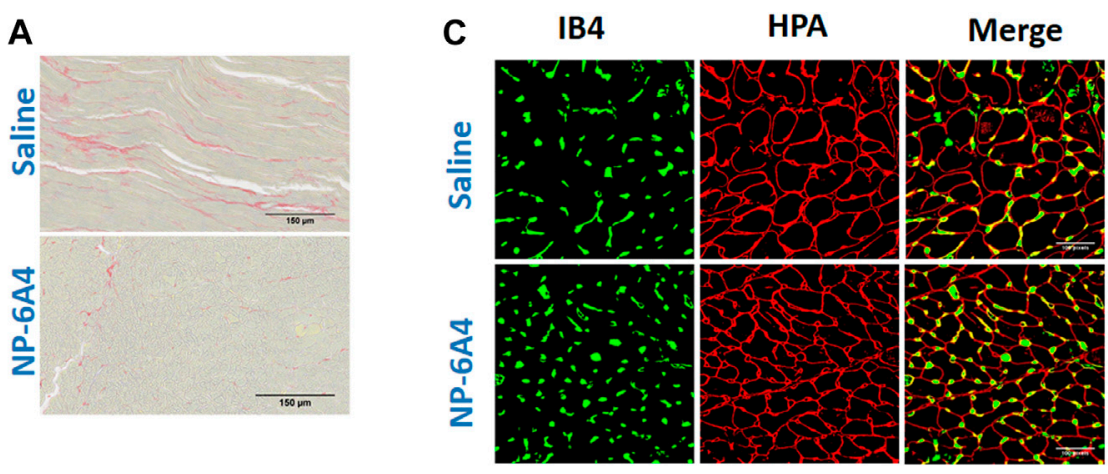

B

D

E
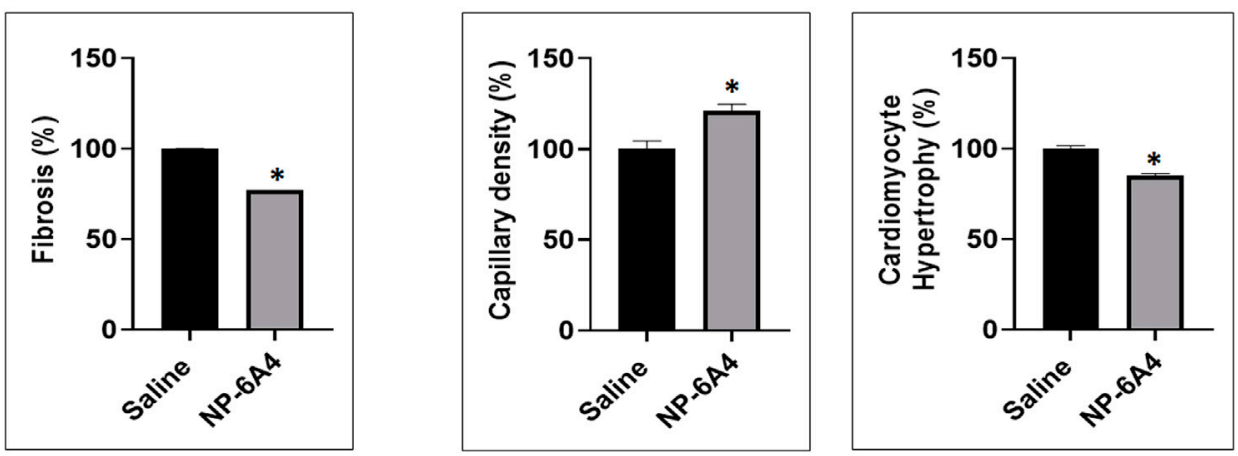

FIGURE 3 | NP-6A4 treatment attenuated cardiac fibrosis, hypertrophy and loss of cardiac microvascular density in ZO rats with untreated diabetes and obesity.

(A) Representative images of PSR stained heart sections of ZO rats treated with saline or NP-6A4 taken at $\times 20$ magnification (scale bars $=150 \mu \mathrm{m}$ ). (B) Graph shows the cumulative data for normalized fibrotic square area ( $n=6$ animals per group; 10 images per animal; 60 images per group). (C) Representative images of heart sections co-stained with HPA-Alexa Fluor (red) to visualize cardiomyocyte membrane IB4-Alexa Fluor 594 (green) to visualize capillaries ( $\times 40$ magnification; scale bars = $100 \mu \mathrm{m}$ ). (D and E) Graphs show cumulative data for cardiomyocyte size and the number of capillaries per square area. $n=6$ animals per group; 10 images per animal; and 65 cells per animal from multiple images.

consumption (Duscha et al., 1999). It is also shown that chronic diabetes induces loss of capillary density (capillary rarefaction) and that diabetic human myocardial explants exhibit significant capillary rarefaction and pericyte loss compared to nondiabetic explants (Hinkel et al., 2017). Therefore, we tested whether NP-6A4 treatment modulated cardiac capillary density in obese and prediabetic $\mathrm{ZO}$ rats. Co-staining of the HPA-stained sections with the vascular stain Isolectin B4 (IB4) to determine capillary presence showed that NP-6A4 treatment increased IB4 staining by $20.8 \%$ suggesting that NP-6A4 increased cardiac capillary density in ZO rats (Figures 3C,E). Given the significance of $20 \%$ capillary rarefaction in muscle pathology of diabetic patients, NP-6A4mediated increase of $20.8 \%$ cardiac capillary density in $\mathrm{ZO}$ rats with untreated obesity and pre-diabetes is likely a significant contributor to their improved cardiac function in response to NP-6A4-treatment. Moreover, increase in capillary density is consistent with NP-6A4-induced improvement of human coronary endothelial cell function (Toedebusch et al., 2018).

\section{NP-6A4 Activated a Feed-Forward Loop That Increased Cardiac AT2R Expression}

AT2R agonists CGP42112A and C21 do not increase AT2R expression in the heart and are not used to treat heart disease
(Wan et al., 2004; Chow et al., 2016). We reported that NP-6A4 increased AT2R expression in human cardiovascular cells (Toedebusch et al., 2018). Consistent with this, NP-6A4treatment increased Agtr2 mRNA expression by at least 5fold in $\mathrm{ZO}$ rat hearts as assessed by quantitative RT-PCR (Figure 4A). Immunohistochemistry analysis using antiAT2R-antibody also showed an increase in AT2R protein expression in NP-6A4-treated $\mathrm{ZO}$ rat hearts (Figures 4B,C). However, protein expression levels of other RAAS components (Gul et al., 2012) such as AT1 receptor subtypes AT1A (Figure 4D) and AT1B (Figure 4E), or anti-inflammatory RAAS components such as MAS receptor (Figures $4 \mathbf{F}, \mathbf{G}$ ) and ACE2 (Figures $\mathbf{4 H}, \mathbf{I}$ ) did not change in response to NP$6 \mathrm{~A} 4$ treatment in $\mathrm{ZO}$ rat hearts.

\section{NP-6A4 Induced Suppression of a Large Array of Inflammatory and Pro-fibrotic Cytokines and Chemokines in ZO Rat Heart}

To gain a better understanding of the mechanisms underlying the cardioprotective effects of NP-6A4-AT2R signaling we analyzed the intracardiac cytokine profile using the Quantibody ${ }^{\circledR}$ Rat Cytokine Array 67 analysis of RayBiotech. Inc. (performed by RayBiotech). Twenty four cytokines were significantly differentially expressed in NP- 

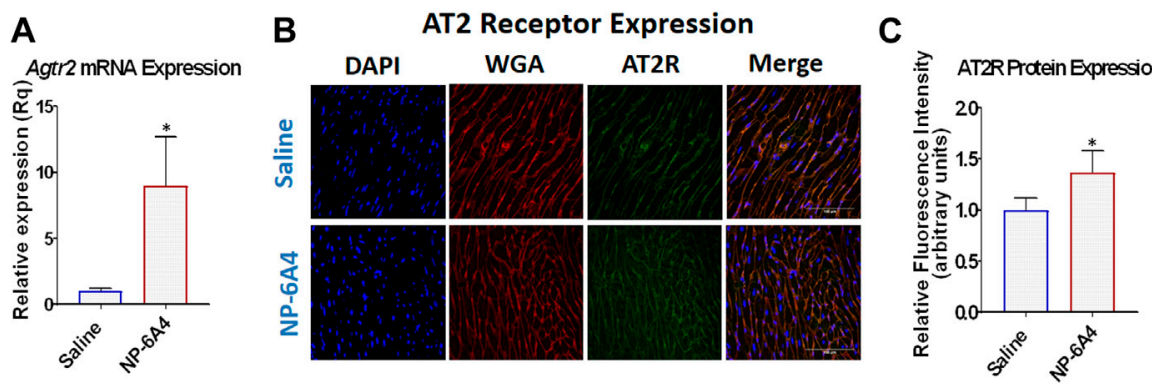

D

E

AT1a mRNA expression AT1b mRNA expression
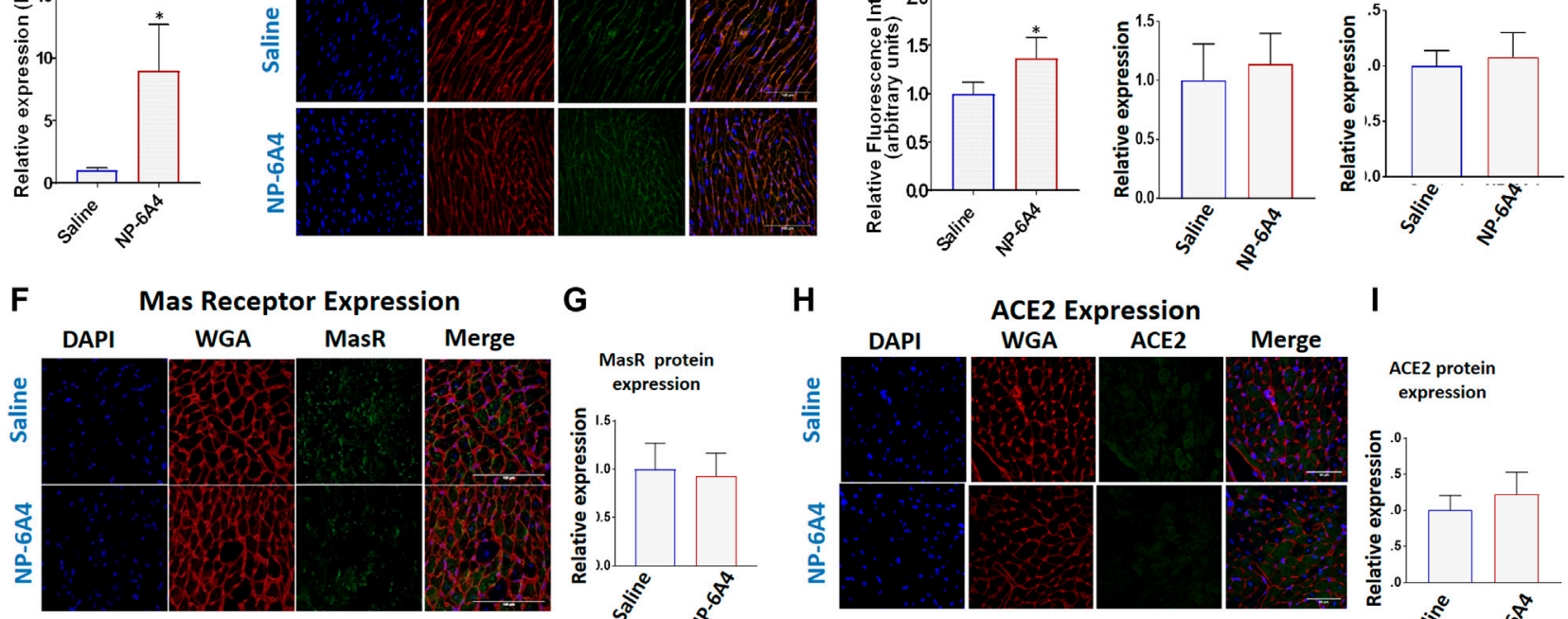

G

H
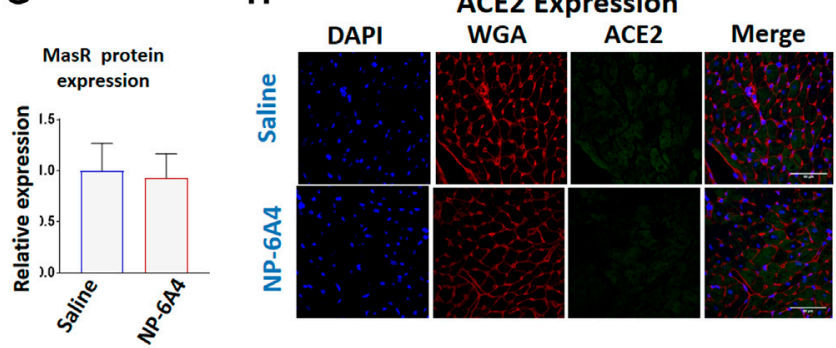

I

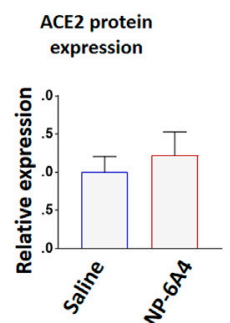

FIGURE 4 | NP-6A4 treatment increased expression of cardiac AT2R, but not AT1 receptor subtypes, Mas receptor or ACE2. (A) Expression of cardiac Agtr2 (AT2R) mRNA as determined by qRT-PCR in Saline-and NP-6A4 treated rats. $n=6$ per group. (B). Representative images of heart sections from immunohistochemistry analysis using anti-AT2R antibody as the probe (green), nuclei visualized by DAPI staining (blue) and cell membranes visualized by staining with WGA-Alexa Fluor 647 (red) ( $\times 63$ magnification, scale bars $=50 \mu \mathrm{m})$. (C) Graphs show cumulative data for normalized anti-AT2R antibody staining. (D and E) Expression of cardiac AT1 receptor subtypes AT1a and AT1b mRNA expression as determined by qRT-PCR. (F and $\mathbf{H})$ Representative images of heart sections from immunohistochemistry analysis using anti-MasR antibody or anti-ACE2 antibody as the probe (green), nuclei visualized by DAPI staining (blue) and cell membranes visualized by staining with WGA-Alexa Fluor 647 (red). (G and I) Graphs show cumulative data for normalized anti-MasR antibody and anti-ACE2 antibody staining.

6A4-treated compared to saline-treated $\mathrm{ZO}$ rat hearts (Figure $\mathbf{5 A}$ ). Among them were 19 pro-inflammatory and pro-fibrotic cytokines indicating that NP-6A4 activates a unique anti-inflammatory signaling that coordinately suppresses several inflammatory cytokines that contribute to the complex cardiac pathology caused by obesity and pre-diabetes. Table 1 shows the roles of interleukins IL-1 $\alpha$ and $\beta$, IL-2, IL-4, IL-6, and IL-13, Fractalkine, Interferon- $\gamma$ (IFN- $\gamma$ ), Tumor necrosis factor- $\alpha$ (TNF- $\alpha)$, Monocyte chemotactic protein-1 (MCP-1), Galactin-3, Granulocyte-monocyte-colony stimulating factor (GM-CSF), Cytokine-induced neutrophil chemoattractant 1 (CINC-1, also known as CXCL1), and Intracellular Adhesion Molecule 1(ICAM-1), in cardiac pathology and are established from clinical and pre-clinical studies. These cytokines are specifically implicated in fibrosis, hypertrophy, cardiomyopathy, coronary heart disease, or heart failure in humans and animal models. LIX, also known as CXCL5, is increased in autoimmune diseases. As shown in Figure 5A, all of these inflammatory cytokines are significantly suppressed by NP-6A4 treatment in $\mathrm{ZO}$ rat heart. Interestingly, these same cytokines are also implicated in cytokine storm induced by infectious diseases including COVID-19 and they are drug targets for mitigating cytokine storm-induced tissue damage (Table 1). Additionally, interleukin-10 (IL-10) secreted by cardiac macrophages has a critical role in cardiac fibrosis specifically in heart failure with preserved ejection fraction (HFpEF) (Hulsmans et al., 2018). NP-6A4 treatment suppressed cardiac IL-10 also in ZO rats
(Figure 5A). L-selectin is a cell adhesion molecule that shows increased expression in inflamed vascular tissues and is implicated in leukocyte infiltration in cardiovascular diseases (Weil and Neelamegham 2019). NP-6A4 treatment suppressed cardiac L-selectin levels also (Figure 5A). Plateletderived growth factor A-chain (PDGF AA) that is increased during vascular hypertrophy and COVID-19 (Zhao et al., 2011; Petrey et al., 2021) and Tissue inhibitor of metalloproteinases (TIMP1) that promotes myocardial fibrosis (Takawale et al., 2017) are also suppressed by NP6A4 (Figure 5). Other molecules suppressed by NP-6A4 include TCK1 and Prolactin receptor, however their potential roles in cardiac functions in conditions of obesity and pre-diabetes are yet to be elucidated.

\section{NP-6A4 Treatment Increased the Expression of Cardioprotective Erythropoietin (EPO) and Notch1 in Addition to the Reparative AT2R}

Cardiac levels of two molecules implicated in cardiac repair, Erythropoietin (EPO) (Santhanam et al., 2010; Zafiriou et al., 2014)) and Notch1 (Yu and Song 2014; Zhu et al., 2020; Li et al., 2021) were significantly increased by NP-6A4 in ZO rat heart (Figure 5A). Thus, NP-6A4 increased the expression of at least three cardioprotective molecules in the $\mathrm{ZO}$ rat heart, namely, AT2R, EPO and Notch1. Increase in the expression of these 
A

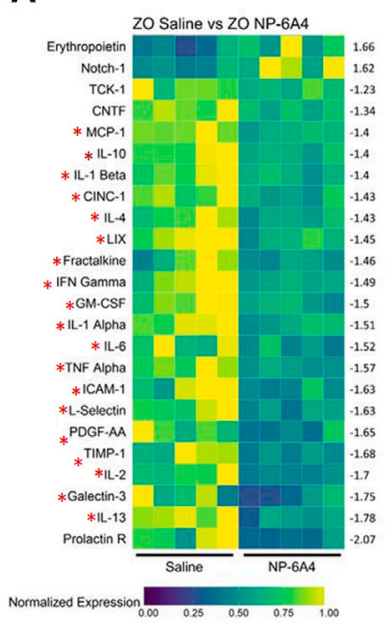

B

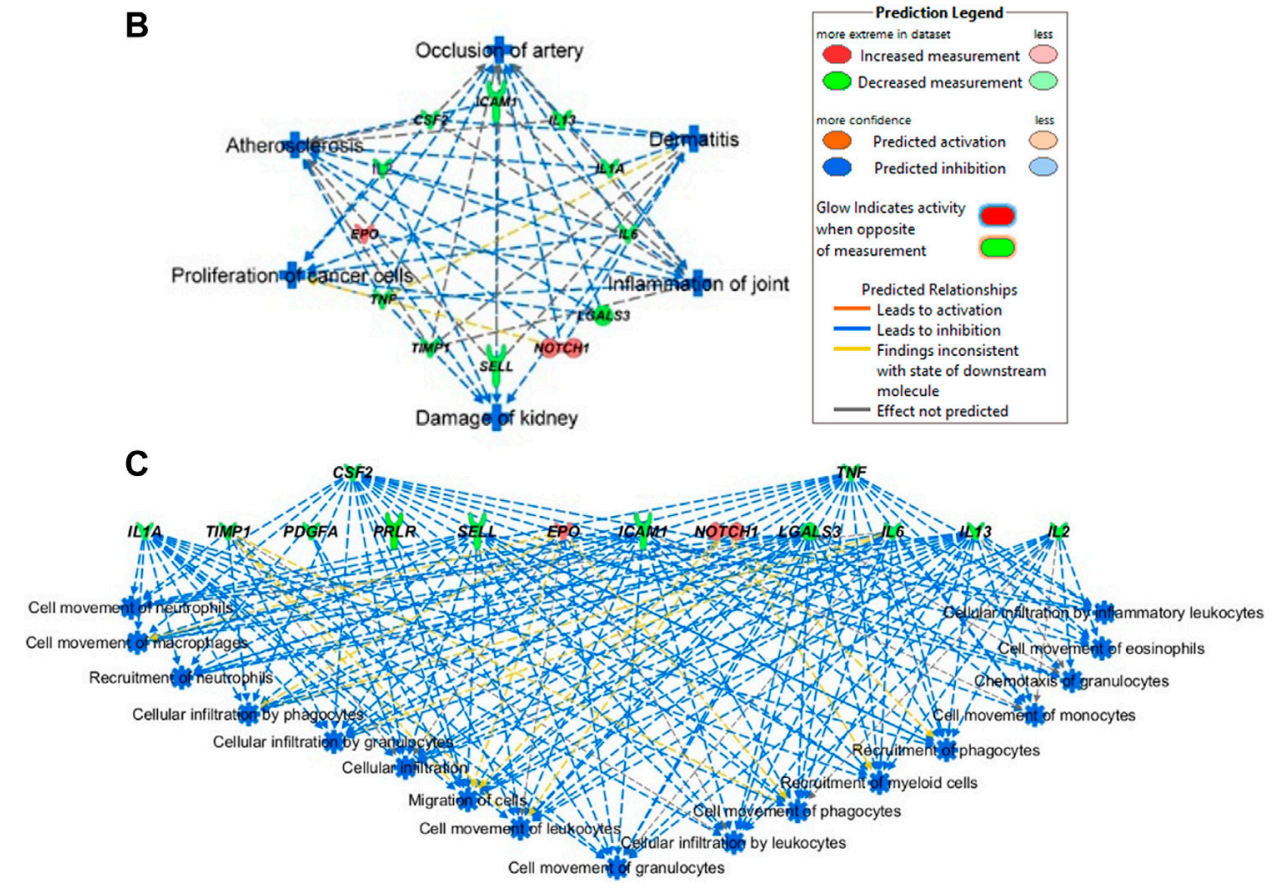

FIGURE 5 | NP-6A4 suppressed a large array of inflammatory and fibrotic cytokines implicated in both cardiac pathologies and cytokine storm and simultaneously increased expression of cardio-protective erythropoietin (EPO) and Notch1 that promote myocardial repair in the heart tissues of obese and pre-diabetic ZO rat. (A) Heat map shows a graphic representation of relative expression of 24 differentially expressed intracardiac cytokines significantly different between saline and NP-6A4 treated ZO rat hearts. Individual cardiac samples are arranged along the $x$-axis and cytokines along the $y$-axis. 19 cardiac molecules implicated in cardiac pathologies (Table 1) and suppressed by NP-6A4 are marked by red star. Fifteen cytokines that exhibited 1.5-fold differential expression in either direction were further selected for IPA analysis (see Supplementary Table S3). $n=5$ animals per group. Yellow indicates the highest expression for each cytokine (1.0), while dark blue indicates lower expression relative to the sample with the highest expression. Expression was normalized for each cytokine across all animals (across each row). Statistical significance was determined using Student's t-test. $p<0.05$ for all proteins. (B) Top scoring IPA-predicted diseases and functions networks resulting from the comparison of cytokine proteins (Supplementary Table S3) of saline and NP-6A4 treated ZO rat hearts. Six diseases and functions were predicted to be suppressed (blue in color) by NP-6A4 treatment based on this cytokine profile. Additional details for each individual network in this group are provided in Supplementary Figure S2. (C) Immune cell specific functions that are predicted to be suppressed in NP-6A4 treated ZO rat hearts. Green cytokines indicate downregulation in NP-6A4-treated compared with saline-treated ZO rat hearts. The blue connecting lines show a known relationship between the cytokine and the cellular process/function. This figure was generated using IPA's built in feature for "Diseases and Functions", and then selecting "immune system related" processes.

protective molecules combined with suppression of a large array of pro-inflammatory and pro-fibrotic molecules by NP-6A4 indicate that NP-6A4 treatment induces an unprecedented cardioprotective cytokine network in the heart tissues of $\mathrm{ZO}$ rats even in the presence of their untreated obesity and prediabetes.

\section{Ingenuity Pathway Analysis Predicts NP-6A4-AT2R-Induced Cardiac Cytokine Network is Cardiovascular and Renal Protective}

To gain a better insight into how NP-6A4 induced cytokine network modulates cardiovascular pathology, we selected cytokines that had at least 1.5-fold change in either direction (Supplementary Table S3) and used them as input for Qiagen's Ingenuity Pathway Analysis (IPA) software. According to IPA prediction, NP-6A4-induced cytokine network could significantly suppress the following diseases and functions: 1) occlusion of artery, 2) atherosclerosis, 3) damage to kidney, 4) inflammation of joint, 5) proliferation of cancer cells and 6) dermatitis. ( $p>0.001$;
Figure 5B). Details on activation $\mathrm{Z}$ score and number of molecules in each pathway are shown in Supplementary Figure S1). Additionally, IPA predicted significant suppression of inflammatory response by NP-6A4 in ZO rat heart (Figure 5C). These results of IPA analysis further suggest that there is activation of a unique cardioprotective and anti-inflammatory cytokine signature by NP-6A4 in ZO rat heart in conditions of untreated obesity, hyperlipidemia, and T2DM. This is a key mechanism for NP$6 \mathrm{~A} 4$ 's ability to attenuate myocardial functional and structural damage resulting from obesity and pre-diabetes.

\section{Other Systemic Effects of NP-6A4: Suppression of Urinary $\beta$-NAG, Lung Weight Adjusted to Tibia Length, and Plasma Alkaline Phosphatase}

While NP-6A4 treatment did not change the 24-h urine output significantly (Table 2 ), it reduced the total amount of $\mathrm{N}$-acetyl- $\beta$ - glucosamynidase ( $\beta$ - NAG) by $66 \%$ in $24-\mathrm{h}$ urine sample of $\mathrm{ZO}$ rats (Table 2). Urinary $\beta$ - NAG is a 
TABLE 1 | Established roles of cytokines and chemokines suppressed by NP-6A4 in ZO rat heart in different pathologies.

\begin{tabular}{|c|c|c|c|c|c|c|c|c|c|}
\hline \multirow{2}{*}{$\begin{array}{l}\text { Cardiac } \\
\text { cytokines/ } \\
\text { chemokines } \\
\text { suppressed } \\
\text { by NP-6A4 }\end{array}$} & \multicolumn{7}{|c|}{ Increased expression levels in } & \multirow{2}{*}{$\begin{array}{l}\text { Inhibitors } \\
\text { used in } \\
\text { clinic for } \\
\text { heart } \\
\text { disease/ } \\
\text { covID-19 }\end{array}$} & \multirow[t]{2}{*}{ References } \\
\hline & $\begin{array}{l}\text { Myocardial } \\
\text { hypertrophy }\end{array}$ & $\begin{array}{l}\text { Cardiac } \\
\text { fibrosis/ } \\
\text { leukocyte } \\
\text { infiltration }\end{array}$ & $\begin{array}{l}\text { Cardio- } \\
\text { myopathy/ } \\
\text { myocarditis }\end{array}$ & $\begin{array}{l}\text { Myocardial } \\
\text { infarction/ } \\
\text { Heart failure }\end{array}$ & $\begin{array}{l}\text { Obesity/ } \\
\text { Diabetes }\end{array}$ & $\begin{array}{c}\text { Auto- } \\
\text { inflammatory } \\
\text { diseases }\end{array}$ & $\begin{array}{l}\text { Cytokine } \\
\text { storms/ } \\
\text { CoVID-19 }\end{array}$ & & \\
\hline IL-1 $\alpha$ and $\beta$ & Yes & Yes & Yes & Yes & Yes & Yes & Yes & Anakrina & $\begin{array}{l}\text { Abbate et al. (2020); } \\
\text { Van Tassell et al. } \\
\text { (2018); } \\
\text { Conti et al. (2020); } \\
\text { Peiró et al. (2017) }\end{array}$ \\
\hline IL-2 & - & - & Yes & - & Yes & Yes & Yes & - & $\begin{array}{l}\text { Thavendiranathan } \\
\text { et al. (2011); } \\
\text { Mangalmurti and } \\
\text { Hunter. (2020); } \\
\text { Frati et al. (2017) }\end{array}$ \\
\hline IL-4 & - & Yes & Yes & - & Yes & - & Yes & - & $\begin{array}{l}\text { Kanellakis et al. } \\
\text { (2012); } \\
\text { Diny et al. (2017); } \\
\text { Schmidt et al. (2015); } \\
\text { Fara et al. (2020) }\end{array}$ \\
\hline IL-6 & Yes & Yes & Yes & Yes & Yes & Yes & Yes & $\begin{array}{l}\text { Tocilizumab } \\
\text { Sarilumab } \\
\text { Siltuximab }\end{array}$ & $\begin{array}{l}\text { Bacchiega et al. } \\
\text { (2017); Mangalmurti } \\
\text { and Hunter. (2020); } \\
\text { Zhang et al. (2016); } \\
\text { Chen et al. (2020) }\end{array}$ \\
\hline IL-13 & - & Yes & - & - & - & - & Yes & - & $\begin{array}{l}\text { Costela-Ruiz et al. } \\
\text { (2020); Liu et al. } \\
(2015)\end{array}$ \\
\hline Fractalkine & - & Yes & Yes & Yes & Yes & Yes & Yes & $\mathrm{KAND} 67^{\star}$ & $\begin{array}{l}\text { Costela-Ruiz (et al. } \\
\text { (2020); } \\
\text { Xuan et al. (2011); } \\
\text { Kikuchi et al. (2004) }\end{array}$ \\
\hline $\mathrm{IFN}-\gamma$ & - & - & Yes & Yes & Yes & - & Yes & - & $\begin{array}{l}\text { Costela-Ruiz et al. } \\
\text { (2020); } \\
\text { Torzewski et al. } \\
\text { (2012); Reifenberg } \\
\text { et al. } 2007\end{array}$ \\
\hline TNF- $\alpha$ & Yes & Yes & Yes & Yes & Yes & Yes & Yes & $\begin{array}{l}\text { Remicade, } \\
\text { Enbrel, } \\
\text { Humira, } \\
\text { Cimzia, } \\
\text { Simponi }\end{array}$ & $\begin{array}{l}\text { Costela-Ruiz et al. } \\
\text { (2020); } \\
\text { Schumacher and } \\
\text { Naga Prasad (2018) }\end{array}$ \\
\hline MCP-1 & Yes & Yes & Yes & Yes & Yes & Yes & Yes & - & $\begin{array}{l}\text { Costela-Ruiz et al. } \\
\text { (2020); } \\
\text { Rolski and Błyszczuk } \\
\text { (2020); } \\
\text { Niu and Kolattukudy. } \\
\text { (2009) }\end{array}$ \\
\hline Galactin-3 & - & Yes & Yes & Yes & Yes & Yes & Yes & - & $\begin{array}{l}\text { Costela-Ruiz et al. } \\
\text { (2020): } \\
\text { Souza et al. (2017); } \\
\text { Vora et al. (2019); } \\
\text { Caniglia et al. (2020) }\end{array}$ \\
\hline GM-CSF & Yes & Yes & Yes & Yes & Yes & Yes & Yes & Lenzilumab & $\begin{array}{l}\text { Costela-Ruiz et al. } \\
\text { (2020): } \\
\text { Anzai et al. (2017) }\end{array}$ \\
\hline CINC1 (CXCL1) & Yes & Yes & Yes & Yes & Yes & Yes & Yes & - & $\begin{array}{l}\text { Costela-Ruiz et al. } \\
\text { (2020): } \\
\text { Zagorski et al. (2007); } \\
\text { Wang et al. (2018) }\end{array}$ \\
\hline ICAM1 & Yes & Yes & Yes & Yes & Yes & Yes & Yes & $\begin{array}{l}\text { Xiidra } \\
\text { (lifitegrast) }\end{array}$ & $\begin{array}{l}\text { Costela-Ruiz et al. } \\
\text { (2020): } \\
\text { Salvador et al. (2016); } \\
\text { Luc et al. (2003) } \\
\text { d on following page) }\end{array}$ \\
\hline
\end{tabular}


TABLE 1 | (Continued) Established roles of cytokines and chemokines suppressed by NP-6A4 in ZO rat heart in different pathologies.

\begin{tabular}{|c|c|c|c|c|c|c|c|c|c|}
\hline \multirow{2}{*}{$\begin{array}{l}\text { Cardiac } \\
\text { cytokines/ } \\
\text { chemokines } \\
\text { suppressed } \\
\text { by NP-6A4 }\end{array}$} & \multicolumn{7}{|c|}{ Increased expression levels in } & \multirow{2}{*}{$\begin{array}{l}\text { Inhibitors } \\
\text { used in } \\
\text { clinic for } \\
\text { heart } \\
\text { disease/ } \\
\text { covID-19 }\end{array}$} & \multirow[t]{2}{*}{ References } \\
\hline & $\begin{array}{l}\text { Myocardial } \\
\text { hypertrophy }\end{array}$ & $\begin{array}{c}\text { Cardiac } \\
\text { fibrosis/ } \\
\text { leukocyte } \\
\text { infiltration }\end{array}$ & $\begin{array}{l}\text { Cardio- } \\
\text { myopathy/ } \\
\text { myocarditis }\end{array}$ & $\begin{array}{l}\text { Myocardial } \\
\text { infarction/ } \\
\text { Heart failure }\end{array}$ & $\begin{array}{l}\text { Obesity/ } \\
\text { Diabetes }\end{array}$ & $\begin{array}{c}\text { Auto- } \\
\text { inflammatory } \\
\text { diseases }\end{array}$ & $\begin{array}{c}\text { Cytokine } \\
\text { storms/ } \\
\text { COVID-19 }\end{array}$ & & \\
\hline LIX (CXCL-5) & - & - & - & - & - & Yes & Yes & - & $\begin{array}{l}\text { Costela-Ruiz et al. } \\
\text { (2020): } \\
\text { Koenig et al. (2020) }\end{array}$ \\
\hline IL-10 & - & Yes & - & Yes & Yes & - & Yes & - & $\begin{array}{l}\text { Hulsmans et al. } \\
(2018)\end{array}$ \\
\hline L-Selectin & - & Yes & - & - & & - & $?$ & & $\begin{array}{l}\text { Weil and } \\
\text { Neelamegham. } \\
\text { (2019) }\end{array}$ \\
\hline PDGF-AA & - & Yes & Yes & Yes & Yes & - & Yes & - & $\begin{array}{l}\text { Zhao et al. (2011); } \\
\text { Petrey et al. (2021) }\end{array}$ \\
\hline TIMP-1 & - & Yes & - & - & - & - & - & - & Takawale et al. (2017) \\
\hline
\end{tabular}

marker for tubulointerstitial injury in diabetes (Kim et al., 2016). Nephroprotective treatments such as with acetaminophen is shown to reduce $\beta$ - NAG in the urine of $\mathrm{ZO}$ rats by $41 \%$ (89). Therefore, our observation that NP-6A4 reduced urinary $\beta$ - NAG in a 24 -h urine sample indicates that $\mathrm{NP}-6 \mathrm{~A} 4$ treatment is nephroprotective in $\mathrm{ZO}$ rats. NP-6A4 also induced a modest, but significant reduction in total enzymatic creatinine in the 24 -h urine sample (Table 2). Other urine metabolites (sodium, potassium, glucose, Gamma-Glutamyl-Transferase, and protein) did not change significantly (Table 2). Additionally, as shown in Figure 2; Table 2, heart, kidney and liver wet weights adjusted to tibia length were not significantly different between $\mathrm{ZO}$ rats treated with saline or NP-6A4. However, wet weight of lung was reduced by $15 \%(p=0.023)$ in response to NP-6A4 in ZO rats (Table 2). This observation indicated that NP-6A4 treatment modulated lung weight in an animal model that exhibits diastolic dysfunction with preserved ejection fraction, obesity and pre-diabetes. Lung congestion is associated with chronic heart failure
(Melenovsky et al., 2015). However, additional studies are needed to fully evaluate the role of NP-6A4 on lung complications induced by obesity and chronic inflammation. $\mathrm{ZO}$ rats are reported to have elevated plasma alkaline phosphatase, a marker of liver and bone injury (Gary-Bobo et al., 2007). NP-6A4 treatment significantly reduced fasting plasma alkaline phosphatase (Figure 2M) indicating that NP-6A4 treatment may be protective for liver and bone tissues.

\section{NP-6A4 Increased miRNAs That Inhibits Cardiac Fibrosis and Hypertrophy and Suppress Inflammatory Cytokines}

MicroRNAs have emerged as powerful diagnostic markers for heart disease and master regulators of gene expression. Therefore, we analyzed NP-6A4-induced changes in miRNA expression pattern in $\mathrm{ZO}$ rat hearts. Analysis of miRNA expression patterns using the Affymetrix miRNA GeneChip Version 4.0 identified 95 miRNAs that were significantly

TABLE 2 | Effects of NP-6A4 treatment on urine parameters and wet weights of other tissues than heart in Zucker obese rats with untreated diabetes, obesity, and metabolic syndrome.

\begin{tabular}{|c|c|c|c|}
\hline Treatment & Saline & NP-6A4 & $p$ value \\
\hline \multicolumn{4}{|l|}{ Metabolites (total amount in $\mathbf{2 4} \mathrm{h}$ urine) } \\
\hline 24-h urine (ml) & $36 \pm 6$ & $41 \pm 6$ & 0.45 \\
\hline $\mathrm{Na}^{+}(\mathrm{mEq})$ & $3.4 \pm 0.4$ & $3.0 \pm 0.3$ & 0.62 \\
\hline $\mathrm{K}^{+}(\mathrm{mEq})$ & $7.8 \pm 1.07$ & $5.25 \pm 0.46$ & 0.08 \\
\hline Glucose (mg) & $1,433 \pm 486$ & $1,343 \pm 372$ & 0.53 \\
\hline Gamma-glutamyl transferase (GGT) (U) & $12.11 \pm 3.8$ & $12.09 \pm 2.2$ & 0.92 \\
\hline $\mathrm{N}$-acetyl- $\beta$-glucosamynidase ( $\beta$-NAG) (mU) & $879.2 \pm 128$ & $385.3 \pm 99$ & 0.017 \\
\hline Protein $(\mathrm{mg})$ & $412 \pm 130$ & $191 \pm 48$ & 0.07 \\
\hline Enzymatic creatinine & $219.5 \pm 24$ & $86.6 \pm 8.5$ & 0.0006 \\
\hline
\end{tabular}

Wet tissue weights (gram) adjusted to tibia length (cm)

\begin{tabular}{|c|c|c|c|}
\hline Lung & $0.337 \pm 0.06$ & $0.286 \pm 0.06$ & 0.02 \\
\hline Liver & $7.11 \pm 0.58$ & $6.08 \pm 0.55$ & 0.16 \\
\hline Kidney & $1.06 \pm 0.014$ & $0.88 \pm 0.15$ & 0.056 \\
\hline
\end{tabular}



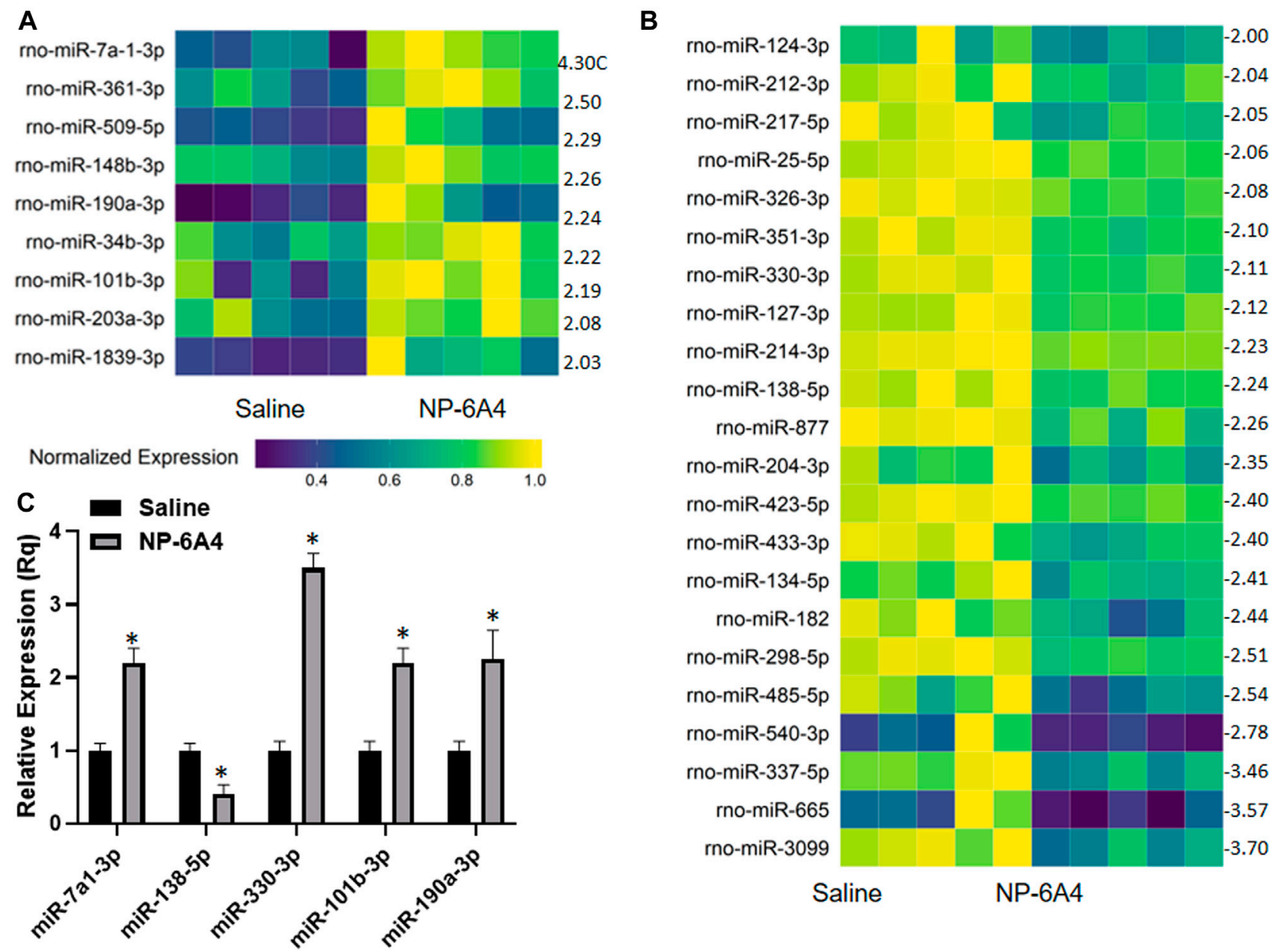

FIGURE 6 |NP-6A4 modulates a cardiac microRNAs that can potentially regulate the anti-inflammatory protein network in ZO rat heart. (A and B) Heat maps show graphic representation of relative expression of intracardiac microRNAs that exhibited at least a two-fold increase (A) or two-fold decrease (B) in NP-6A4-vs. salinetreated ZO rat hearts. $n=5$ animals per group. Yellow indicates the highest expression for each cytokine (1.0), while dark blue indicates lower expression relative to the sample with the highest expression. Expression was normalized for each microRNA across all animals (across each row). Statistical significance was determined using Student's $t$-test. $p<0.05$ for all differentially expressed miRNAs. Supplementary Tables S4A,B list all miRNAs that exhibited at least 1.5-fold differential expression. (C) Graph shows the results of quantitative RT-PCR analysis of some of the differentially expressed miRNAs in NP-6A4 vs. saline treated ZO rat hearts previously identified by microarray analysis. $n=6$ animals per group and qRT-PCR was performed in triplicates. ${ }^{*} p<0.05$ for saline-treated vs. NP-6A4 treated as determined by two-tailed paired $t$-test.

differentially expressed by at least 1.5 -fold (Supplementary Tables S4A,B), of which 31 miRNAs showed a 2-fold differential expression (Figures 6A,B). Quantitative RT-PCR analysis was used to spot check the expression patterns of some of these miRNAs as an alternative method to verify the data from microarray. All miRNAs tested by qRT-PCR confirmed the differential expression pattern seen in microarray analysis (Figure 6C). Next we checked whether any of the miRNAs that showed increased expression were reported to have cardioprotective effects according to literature. We found that miR-101b-3p abrogates cardiomyocyte hypertrophy and fibrosis (Pan et al., 2012; Lee et al., 2017) and miR-148b-3p, is downregulated in mitral regurgitation and heart failure (Chen et al., 2016; Figure 6A; Table 3). Literature also showed that miR-101b-3p suppresses Stc1 (Stanniocalcin 1) implicated in macrophage differentiation, preferentially M1 polarization, and contributes to the increase in expression of multiple inflammatory cytokines (Table 3; De Martino et al., 2009; Leung and Wong, 2021) Similarly, miR-190a-3p is shown to target and suppress Tiam1 that activates ill7 promoter and increase inflammatory cytokines (Table 3; Kurdi et al., 2016. Liu et al., 2019). It is conceivable that up-regulation of miRNAs that suppress different positive regulators of inflammatory cytokines can be one potential mechanism underlying this coordinated suppression of a large array of inflammatory cytokines by NP-6A4 (Figure 7). However, additional studies are needed to test this idea.

\section{DISCUSSION}

Results presented here show for the first time that NP-6A4, a peptide agonist of AT2R with an FDA designation for pediatric 
TABLE 3 | Differentially expressed cardiac miRNAs of ZO rats in response to NP-6A4 treatment may contribute to mitigating cardiac damage, suppressing inflammatory cytokines and regulating expression of AT2R, EPO and Notch1.

\begin{tabular}{|c|c|c|}
\hline miRNA & $\begin{array}{l}\text { Fold change in } \\
\text { NP-6A4 treated rat } \\
\text { hears as per } \\
\text { microarray analysis }\end{array}$ & Confirmed and/or predicted function \\
\hline miR-148-b3p & 2.2 & Associated with decrease in mitral regurgitation Chen et al. (2016) \\
\hline miR-101b-3p & 2.18 & $\begin{array}{l}\text { Associated with decrease in cardiac fibrosis and hypertrophy Pan et al. (2012); Lee et al. (2017). Targets and suppresses } \\
\text { Stc1 implicated in M1 macrophage polarization and increasing expression of inflammatory cytokines De Martino et al. } \\
\text { (2009); Leung and Wong (2021) }\end{array}$ \\
\hline miR-190a-3p & 2.23 & Shown to suppress Tiam1 that increases expression of inflammatory cytokines Kurdi et al. (2016); Liu et al. (2019) \\
\hline miR-182 & -2.43 & Decreased expression $\rightarrow$ increased expression of EPO, Notch1, and EphA5 ${ }^{a}$ \\
\hline miR-138-5p & -2.23 & Decreased expression $\rightarrow$ increased expression of AT2R, EPO, and Notch1 ${ }^{a}$ \\
\hline miR-330-3p & -2.1 & Decreased expression $\rightarrow$ increased expression of AT2R and Notch $1^{a}$ \\
\hline miR-337-5p & -3.45 & Decreased expression $\rightarrow$ increased expression of EphA5 ${ }^{a}$ \\
\hline
\end{tabular}

${ }^{a}$ Denotes predicted effects of these miRNA changes on the expression of cardiac proteins based on the in silico analysis using miRDB software.

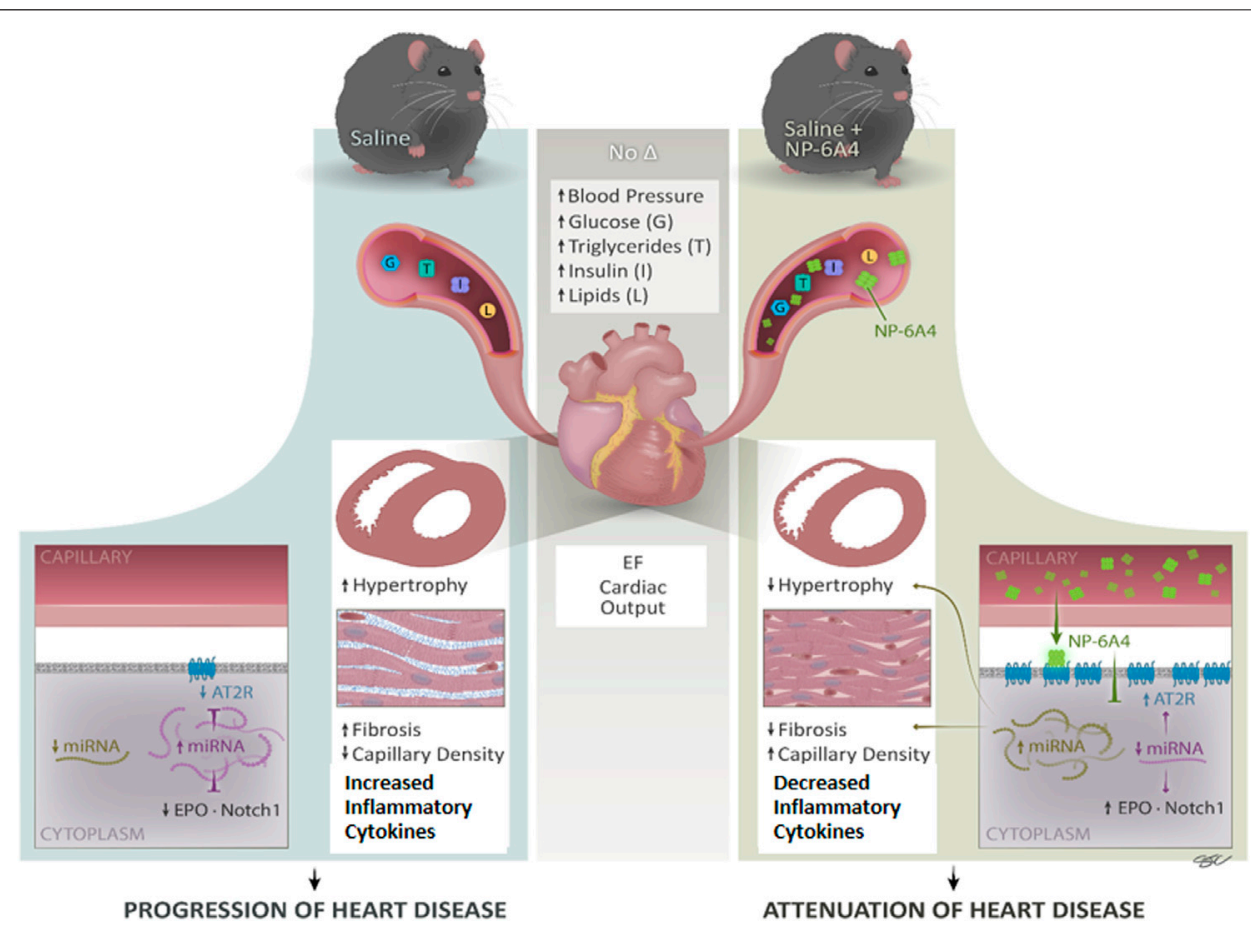

FIGURE 7 | NP-6A4 mitigates heart disease via a new multi-prong signaling that coordinates expression of cardioprotective protein and miRNA in conditions of untreated obesity and pre-diabetes. Cartoon diagram summarizes the key events underlying cardioprotection induced by NP-6A4-AT2R signaling in untreated diabetes and obesity. NP-6A4 treatment attenuated cardiac dysfunction as evidenced by reduced E/e', improved GLS and GCS. NP-6A4 suppressed myocardial structural damage as evidenced by reduction in fibrosis, cardiomyocyte hypertrophy, and loss of microvascular density. These protective effects were mediated by a coordinated increase in the expression of cardio-reparative AT2R, EPO and Notch1 (marked in the diagram) and suppression of nineteen pro-inflammatory and/or profibrotic cytokines. Expression of multiple miRNAs that can potentially target AT2R, EPO and Notch1 (shown in purple) are suppressed whereas miRNAs implicated in attenuating heart failure, hypertrophy and fibrosis and suppressing inflammatory cytokines (shown in light green) are increased by NP-6A4-AT2R signaling. Since all these cardioprotective effects are induced in conditions of untreated obesity, and without modulating blood pressure, NP-6A4 can be an exciting synergistic drug for anti-hyperglycemic, anti-lipidemic and anti-hypertensive drugs to mitigate severe cardiac inflammation and heart disease.

cardiomyopathy, could suppress an inflammatory cytokine network comprised of 21 proteins in the heart tissues of $\mathrm{ZO}$ rats with untreated obesity and T2DM, and ameliorate cardiac structural and functional damage. Previous studies have shown that the presence of either a high $\mathrm{E} / \mathrm{E}^{\prime}$ ratio or an impaired myocardial global longitudinal strain (GLS) provides a useful negative predictive value of cardiovascular events in patients with type 2 diabetes (Liu et al., 2016).

We show that in young obese and pre-diabetic $\mathrm{ZO}$ rats that exhibit cardiac dysfunction with preserved ejection fraction, NP6A4 treatment attenuated early myocardial deformation [as evidenced by improvement of myocardial GLS and 
circumferential strain (GCS)], and diastolic dysfunction (as evidenced by reduction in E/e' and MPI). NP-6A4 also suppressed cardiac fibrosis and cardiomyocyte hypertrophy, and increased cardiac capillary density in $\mathrm{ZO}$ rats. The mode of action of NP-6A4 involves activating a positive feedback loop that increases expression of its receptor, the cardio-reparative AT2R. However, unlike AT1 receptor blockers (Igase et al., 2008), and other AT2R agonists (Sumners et al., 2015; Dai et al., 2016), NP-6A4 did not increase cardiac levels of ACE2 protein indicating that NP-6A4-AT2R-signaling is unique. Mas receptor protein levels in the heart were also unaffected by NP-6A4.

NP-6A4 treatment did not induce any significant changes in bodyweight, fasting plasma glucose, insulin, triglycerides, and cholesterol, indicating that cardioprotective effects of NP-6A4 did not stem from modulating these parameters. Although central activation of $\mathrm{AT} 2 \mathrm{R}$ is involved in reducing blood pressure, systemically applied AT2R agonists do not change blood pressure and AT2R agonists are not used as anti-hypertensive drugs (Sumners et al., 2015; Dai et al., 2016). Consistent with this, we also did not see any significant changes in blood pressure in $\mathrm{ZO}$ rats in response to this sub-cutaneous NP-6A4 treatment (Supplementary Table S2). Thus, cardioprotective effects of NP$6 \mathrm{~A} 4$ do not arise from significant blood pressure reduction. These observations suggest that NP-6A4 can be cardioprotective in the continuous presence of a toxic metabolic profile indicated by hyperglycemia, hyperinsulinemia, and hyperlipidemia.

Capillary loss or rarefaction is a dangerous cardiac pathology observed in human diabetic hearts and skeletal muscle biopsies of patients with chronic heart failure (Duscha et al., 1999; Hinkel et al., 2017). We have reported previously that NP-6A4 increased endothelial function as evidenced by increased expression of endothelial nitric oxide synthase and generation of nitric oxide in human coronary artery endothelial cells (Toedebusch et al., 2018). Therefore, the observed increase in cardiac capillary density in $\mathrm{ZO}$ rats could be the result of improved endothelial function induced by NP-6A4-AT2R signaling in $\mathrm{ZO}$ rat heart. Such improvement in capillary density is key to improved cardiac function.

Our data show that NP-6A4-AT2R signaling suppresses 19 cardiac cytokines and chemokines involved in pathologic remodeling of the heart (Figures 4A-C). Among them are the IL-1 ligands, IL- $1 \alpha$ and $\beta$, that act through IL-1 receptor, and implicated in NRLP3 inflammasome-induced cardiac inflammation, diabetic cardiomyopathy, septic shock, myocardial infarction, heart failure and atherosclerosis (Peiró et al., 2017; Abbate et al., 2020). IL-1 receptor blockers or monoclonal antibodies against IL- $1 \beta$ are currently in clinical trials to mitigate cardiac inflammation. Moreover, Anakrina, an IL-1 receptor blocker, is in clinical trials to mitigate cardiac and pulmonary inflammation and death (Cauchois et al., 2020; Chen et al., 2020). IL-2 implicated in myocarditis, and IL4, IL-6, and IL-13 implicated in fibrosis and cardiomyopathy are the other inflammatory interleukins suppressed by NP-6A4 (Table 1). Fractalkine and Galactin-3 are two other inflammatory cytokines that are implicated in cardiomyopathy and heart failure. Other prominent inflammatory cytokines suppressed by NP-6A4 are IFN $\gamma$, TNF- $\alpha$, MCP-1, GM-CSF, CINC-1 (CXCL-1), ICAM-1, LIX (CXCL1), and
PDGF-AA. These data suggest that NP-6A4-AT2R signaling activates a unique regulation of the large array of pro-inflammatory cytokines implicated in pathologic remodeling of cardiovascular tissues in response to metabolic and infectious diseases.

At the same time NP-6A4-AT2R signaling increases expression of Notch1 and EPO that promote cardiac repair. Notch1 signaling is activated in response to myocardial injury and is involved in suppression of cardiomyocyte apoptosis, cardiac repair and regeneration (Li et al., 2010; Yu and Song, 2014; Zhu et al., 2020). Erythropoietin (EPO) increases myocardial performance and induces cardiac repair after myocardial infarction (Santhanam et al., 2010; Zafiriou et al., 2014). Thus, NP-6A4-AT2R signaling induces a coordinated anti-inflammatory cytokine-network that mitigates cardiac pathologic remodeling in $\mathrm{ZO}$ rats with untreated obesity and pre-diabetes.

To gain insight into the relationship between the NP-6A4induced increase in cardioprotective AT2R, EPO and Notch1 and the changes in intracardiac miRNAs, we performed an in silico analysis. Interestingly, analysis of 3 'untranslated regions of mRNAs coding for human AT2R, EPO, and Notch1 using RegRNA 2.0 software (Chang et al., 2013) showed that multiple miRNAs that are suppressed by NP-6A4 treatment (Figure 6B) had binding sites on the these mRNAs (Table 3; Supplementary Table S5). In contrast, none of the miRNAs that showed increased expression over two-fold in NP-6A4treated hearts (Figure 6A) had binding sites in the $3^{\prime}$ untranslated regions of these mRNAs. This observation suggested that NP-6A4 mediated suppression of some microRNAs can be one of the underlying mechanisms for the NP-6A4-induced increases in the expression of AT2R, EPO and Notch1 in ZO rat heart. Additional studies are warranted to test this idea.

At the age of 9 weeks, $\mathrm{ZO}$ rat heart exhibits diastolic dysfunction, cardiac fibrosis, abnormal cardiomyocyte histoarchitecture, and increased levels of 3-nitrotyrosine, and NADPH oxidase-dependent superoxide compared to Zucker lean rat heart (Zhou et al., 2010). Moreover, another study showed that at the age of 12 -weeks, $\mathrm{ZO}$ rat heart exhibits increased levels of lipid-aldehyde 4-hydroxynonenal (4-HNE), platelet endothelial cell adhesion molecule-1 (PECAM-1) and vascular cell adhesion molecule-1 (VCAM-1) and TNF- $\alpha$ compared to lean Zucker rats indicating the highly inflammatory status of the $\mathrm{ZO}$ rat heart (Martinelli et al., 2020). The $\mathrm{ZO}$ rats in this study were 11-week old, an age when cardiac inflammation and damage caused by untreated obesity and pre-diabetes was progressing. NP-6A4-AT2R signaling seems to have mitigated this cardiac inflammation by activating a unique anti-inflammatory cytokine-miRNA network that suppressed 19 pro-inflammatory and pro-fibrotic molecules and increased cardio-reparative molecules (AT2R, EPO and Notch1) as shown in Figure 7. While the change in expression of each individual molecule was modest in response to NP-6A4 treatment in $\mathrm{ZO}$ rat heart, the coordinated change in their expression that results in an antiinflammatory network underlies the powerful anti-inflammatory mode of action of NP-6A4. This is the key mechanism that causes 
the observed multifactorial improvement in cardiac structure and function (attenuation of hypertrophy and fibrosis, improvement of GLS, GCS, E/e', MPI and cardiac capillary density) in response to NP-6A4 treatment in $\mathrm{ZO}$ rats. To our knowledge, this is the first report that defines an AT2Rinduced intracardiac anti-inflammatory cytokine-miRNA network (Figure 7) with cardiovascular and renal protective effects (as predicted by IPA). Suppression of tubulointerstitial injury marker urinary $\beta-\mathrm{NAG}$ in $\mathrm{ZO}$ rats by NP-6A4-AT2R signaling is nephroprotective and is in agreement with the IPA prediction that the anti-inflammatory cytokine network activated by NP-6A4 can potentially suppress kidney damage (Figure 4B).

Additional studies are needed to fully understand the role NP-6A4-mediated suppression of ciliary neurotrophic factor (CNTF) that usually exhibit protective effects on the heart. This is also true for Prolactin receptor and TCK1 (Creatine kinase, testis isozyme), two other molecules suppressed by NP6A4 treatment since their roles in cardiac functions in conditions of untreated obesity and pre-diabetes are not known. Additional studies on NP-6A4-induced structural and functional changes in kidney are also needed to fully understand the impact of NP-6A4 treatment on the prediabetic kidney.

We reported previously that NP-6A4 increases cellular respiration and attenuates doxorubicin-induced increase in reactive oxygen species in human VSMCs and protects them from acute serum starvation better than four beta-adrenergic receptor blockers and an AT1R blocker (Mahmood and Pulakat, 2015; Toedebusch et al., 2018). Moreover, NP-6A4mediated increase in AT2R expression was observed in human VSMCs, and coronary artery and umbilical vein endothelial cells (Toedebusch et al., 2018). Thus, NP-6A4-AT2R signaling renders protective effects on human cardiovascular cells and improves endothelial function by increasing expression of eNOS and nitric oxide. Data presented here show that NP-6A4-induced cardiovascular protective effects also occur in a pre-clinical model for untreated obesity, pre-diabetes, and cardiac dysfunction. Based on our collective in vitro and in vivo data, we propose that NP-6A4 is a novel therapeutic that can be used alone or synergistic with existing therapeutics (e.g., statins, antihyperglycemics and antihypertensives) to treat heart disease presented with preserved ejection fraction, fibrosis and microvascular damage in conditions of obesity and pre-diabetes. It is important to note that patients with obesity and pre-diabetes have an underlying chronic inflammation that makes them highly vulnerable to infectious diseases such as COVID-19, and high morbidity and mortality is seen in both young and old patients with these conditions. It is also noteworthy that while NP-6A4 protected the heart in a pre-clinical model with severe obesity and pre-diabetes (ZO rat), it did not increase expression levels of ACE2, the receptor for SARS-CoV2 in this model. This makes NP-6A4 an ideal adjuvant drug candidate that can provide cardioprotection and mitigate complications arising from severe and/or chronic inflammation in obese and pre-diabetic patients and in pathologies in which increase in ACE2 levels in response to drug treatment is undesirable.

\section{DATA AVAILABILITY STATEMENT}

The original contributions presented in the study are included in the article/Supplementary Material, further inquiries can be directed to the corresponding author.

\section{ETHICS STATEMENT}

The animal study was reviewed and approved by All animal procedures used in this study were approved prior to the beginning of these studies by the Harry S. Truman Memorial Veterans Hospital (HSTMVH) Subcommittee for Animal Safety and University of Missouri IACUC. All animals were cared for in accordance with the Guidelines for the Care and Use of Laboratory Animals (National Institutes of Health publication 85-23).

\section{AUTHOR CONTRIBUTIONS}

LP conceived the project, designed experiments and funded the project. MG developed the structure of NP-6A4. VGD performed Vevo2100 imaging and analysis; SK and SA evaluated Vevo2100 cardiac imaging data. AM and PB performed qRT-PCR. AM performed histopathology AB performed IHC. LP, AM, and MG analyzed cytokine profiles, performed IPA analysis and drafted manuscript. All authors edited the manuscript.

\section{FUNDING}

This work was supported by NIH NHLBI 1R01HL118376-01 LP, and NIH NHLBI 1R01HL138988-01A1 LP and a small grant from Novopyxis Inc. LP, Boston, MA. The funder was not involved in the study design, collection, analysis, interpretation of data, the writing of this article or the decision to submit it for publication.

\section{ACKNOWLEDGMENTS}

Authors greatly appreciate technical support from Ms. Lisa Watkinson and Mr. Terry Carmack in expert animal care and procedures. We also thank Dr. Charles Wiedmeyer of the Comparative Clinical Pathology Services, LLC (CPath) for performing a comprehensive analysis of fasting plasma chemistry reported here. This work was also supported by resources and facilities from Research Services, Harry S. Truman Memorial Veterans Hospital, including the Small Animal Ultrasound Imaging Center.

\section{SUPPLEMENTARY MATERIAL}

The Supplementary Material for this article can be found online at: https://www.frontiersin.org/articles/10.3389/fphar.2021.693167/ full\#supplementary-material 


\section{REFERENCES}

Abbate, A., Toldo, S., Marchetti, C., Kron, J., Van Tassell, B. W., and Dinarello, C. A. (2020). Interleukin-1 and the Inflammasome as Therapeutic Targets in Cardiovascular Disease. Circ. Res. 126 (9), 1260-1280. doi:10.1161/ CIRCRESAHA.120.315937

Altarche-Xifró, W., Curato, C., Kaschina, E., Grzesiak, A., Slavic, S., Dong, J., et al. (2009). Cardiac C-kit+AT2+ Cell Population Is Increased in Response to Ischemic Injury and Supports Cardiomyocyte Performance. Stem Cells 27, 2488-2497. doi:10.1002/stem.171

Anzai, A., Choi, J. L., He, S., Fenn, A. M., Nairz, M., Rattik, S., et al. (2017). The Infarcted Myocardium Solicits GM-CSF for the Detrimental Oversupply of Inflammatory Leukocytes. J. Exp. Med. 214 (11), 3293-3310. doi:10.1084/jem. 20170689

Arnlöv, J., Ingelsson, E., Risérus, U., Andrén, B., and Lind, L. (2004). Myocardial Performance index, a Doppler-Derived index of Global Left Ventricular Function, Predicts Congestive Heart Failure in Elderly Men. Eur. Heart J. 25, 2220-2225. doi:10.1016/j.ehj.2004.10.021

Bacchiega, B. C., Bacchiega, A. B., Usnayo, M. J., Bedirian, R., Singh, G., and Pinheiro, G. D. (2017). Interleukin 6 Inhibition and Coronary Artery Disease in a High-Risk Population: A Prospective Community-Based Clinical Study. J. Am. Heart Assoc. 6 (3), e005038. doi:10.1161/JAHA.116.005038

Belenchia, A. M., Gavini, M. P., Toedebusch, R. G., DeMarco, V. G., and Pulakat, L. (2018). Comparison of Cardiac miRNA Transcriptomes Induced by Diabetes and Rapamycin Treatment and Identification of a Rapamycin-Associated Cardiac MicroRNA Signature. Oxid Med. Cel. Longev. 2018, 8364608. doi:10.1155/2018/8364608

Bennion, D. M., Jones, C. H., Dang, A. N., Isenberg, J., Graham, J. T., Lindblad, F., et al. (2018). Protective Effects of the Angiotensin II AT2 Receptor Agonist Compound 21 in Ischemic Stroke: a Nose-To-Brain Delivery Approach. Clin. Sci. (Lond) 132 (5), 581-593. doi:10.1042/CS20180100

Blomstrand, P., Engvall, M., Festin, K., Lindström, T., Länne, T., Maret, E., et al. (2015). Left Ventricular Diastolic Function, Assessed by Echocardiography and Tissue Doppler Imaging, Is a strong Predictor of Cardiovascular Events, superior to Global Left Ventricular Longitudinal Strain, in Patients with Type 2 Diabetes. Eur. Heart J. Cardiovasc. Imaging 16 (9), 1000-1007. doi:10.1093/ehjci/jev027

Caniglia, J. L., Guda, M. R., Asuthkar, S., Tsung, A. J., and Velpula, K. K. (2020). A Potential Role for Galectin-3 Inhibitors in the Treatment of COVID-19. PeerJ 8, e9392. doi:10.7717/peerj.9392

Cauchois, R., Koubi, M., Delarbre, D., Manet, C., Carvelli, J., Blasco, V. B., et al. (2020). Early IL-1 Receptor Blockade in Severe Inflammatory Respiratory Failure Complicating COVID-19. Proc. Natl. Acad. Sci. U S A 117 (32), 18951-18953. doi:10.1073/pnas.2009017117

Cavalera, M., Wang, J., and Frangogiannis, N. G. (2014). Obesity, Metabolic Dysfunction, and Cardiac Fibrosis: Pathophysiological Pathways, Molecular Mechanisms, and Therapeutic Opportunities. Transl. Res. 164 (4), 323-335. doi:10.1016/j.trsl.2014.05.001

CDC: Centers for Disease Control and Prevention (2021). Adult Obesity Facts. https://www.cdc.gov/obesity/data/adult.html.

CDC: Centers for Disease Control and Prevention (2020). National Diabetes Statistics Report. https://www.cdc.gov/diabetes/data/statistics-report/index. html.

Chang, T. H., Huang, H. Y., Hsu, J. B., Weng, S. L., Horng, J. T., and Huang, H. D. (2013). An Enhanced Computational Platform for Investigating the Roles of Regulatory RNA and for Identifying Functional RNA Motifs. BMC Bioinformatics 14 (Suppl. 2), S4. doi:10.1186/14712105-14-S2-S4

Chen, L. Y. C., Hoiland, R. L., Stukas, S., Wellington, C. L., and Sekhon, M. S. (2020). Confronting the Controversy: Interleukin-6 and the COVID-19 Cytokine Storm Syndrome. Eur. Respir. J. 56 (4), 2003006. doi:10.1183/ 13993003.03006-2020

Chen, M. C., Chang, T. H., Chang, J. P., Huang, H. D., Ho, W. C., Lin, Y. S., et al. (2016). Circulating miR-148b-3p and miR-409-3p as Biomarkers for Heart Failure in Patients with Mitral Regurgitation. Int. J. Cardiol. 222, 148-154. doi:10.1016/j.ijcard.2016.07.179
Choi, E. Y., Rosen, B. D., Fernandes, V. R., Yan, R. T., Yoneyama, K., Donekal, S., et al. (2013). Prognostic Value of Myocardial Circumferential Strain for Incident Heart Failure and Cardiovascular Events in Asymptomatic Individuals: the Multi-Ethnic Study of Atherosclerosis. Eur. Heart J. 34, 2354-2361. doi:10.1093/eurheartj/eht133

Chow, B. S., Koulis, C., Krishnaswamy, P., Steckelings, U. M., Unger, T., Cooper, M. E., et al. (2016). The Angiotensin II Type 2 Receptor Agonist Compound 21 Is Protective in Experimental Diabetes-Associated Atherosclerosis. Diabetologia 59 (8), 1778-1790. doi:10.1007/s00125016-3977-5

Conti, P., Caraffa, A., Gallenga, C. E., Ross, R., Kritas, S. K., Frydas, I., et al. (2020). Coronavirus-19 (SARS-CoV-2) Induces Acute Severe Lung Inflammation via IL-1 Causing Cytokine Storm in COVID-19: a Promising Inhibitory Strategy. J. Biol. Regul. Homeost. Agents 34 (6), 1971-1975. doi:10.23812/20-1-E

Costela-Ruiz, V. J., Illescas-Montes, R., Puerta-Puerta, J. M., Ruiz, C., and Melguizo-Rodríguez, L. (2020). SARS-CoV-2 Infection: The Role of Cytokines in COVID-19 Disease. Cytokine Growth Factor. Rev. 54, 62-75. doi:10.1016/j.cytogfr.2020.06.001

Dai, S. Y., Zhang, Y. P., Peng, W., Shen, Y., and He, J. J. (2016). Central Infusion of Angiotensin II Type 2 Receptor Agonist Compound 21 Attenuates DOCA/ NaCl-Induced Hypertension in Female Rats. Oxid Med. Cel. Longev. 2016, 3981790. doi:10.1155/2016/3981790

De Martino, I., Visone, R., Fedele, M., Petrocca, F., Palmieri, D., Martinez Hoyos, J., et al. (2009). Regulation of microRNA Expression by HMGA1 Proteins. Oncogene 28 (11), 1432-1442. doi:10.1038/onc.2008.495

Diny, N. L., Baldeviano, G. C., Talor, M. V., Barin, J. G., Ong, S., Bedja, D., et al. (2017). Eosinophil-derived IL-4 Drives Progression of Myocarditis to Inflammatory Dilated Cardiomyopathy. J. Exp. Med. 214 (4), 943-957. doi:10.1084/jem.20161702

Duscha, B. D., Kraus, W. E., Keteyian, S. J., Sullivan, M. J., Green, H. J., Schachat, F. H., et al. (1999). Capillary Density of Skeletal Muscle: a Contributing Mechanism for Exercise Intolerance in Class II-III Chronic Heart Failure Independent of Other Peripheral Alterations. J. Am. Coll. Cardiol. 33, 1956-1963. doi:10.1016/s0735-1097(99)00101-1

Fang, L., Murphy, A. J., and Dart, A. M. (2017). A Clinical Perspective of Antifibrotic Therapies for Cardiovascular Disease. Front. Pharmacol. 8, 186. doi:10. 3389/fphar.2017.00186

Fara, A., Mitrev, Z., Rosalia, R. A., and Assas, B. M. (2020). Cytokine Storm and COVID-19: a Chronicle of Pro-inflammatory Cytokines. Open Biol. 10 (9), 200160. doi:10.1098/rsob.200160

FDA US Food and Drug Administration (2017). Search Orphan Drug Designations and Approvals: 7/3. https://www.accessdata.fda.gov/scripts/opdlisting/oopd/ detailedIndex.cfgridkey $=575417$.

Frati, G., Schirone, L., Chimenti, I., Yee, D., Biondi-Zoccai, G., Volpe, M., et al. (2017). An Overview of the Inflammatory Signalling Mechanisms in the Myocardium Underlying the Development of Diabetic Cardiomyopathy. Cardiovasc. Res. 113 (4), 378-388. doi:10.1093/cvr/cvx011

Frisbee, J. C., Goodwill, A. G., Frisbee, S. J., Butcher, J. T., Brock, R. W., Olfert, I. M., et al. (2014). Distinct Temporal Phases of Microvascular Rarefaction in Skeletal Muscle of Obese Zucker Rats. Am. J. Physiol. Heart Circ. Physiol. 307, H1714-H1728. doi:10.1152/ajpheart.00605.2014

Gary-Bobo, M., Elachouri, G., Gallas, J. F., Janiak, P., Marini, P., Ravinet-Trillou, C., et al. (2007). Rimonabant Reduces Obesity-Associated Hepatic Steatosis and Features of Metabolic Syndrome in Obese Zucker Fa/fa Rats. Hepatology 46 (1), 122-129. doi:10.1002/hep.21641

Gul, R., Ramdas, M., Mandavia, C. H., Sowers, J. R., and Pulakat, L. (2012). RASmediated Adaptive Mechanisms in Cardiovascular Tissues: Confounding Factors of RAS Blockade Therapy and Alternative Approaches. Cardiorenal Med. 2, 268-280. doi:10.1159/000343456

Hinkel, R., Howe, A., Renner, S., Ng, J., Lee, S., Klett, K., et al. (2017). Diabetes Mellitus-Induced Microvascular Destabilization in the Myocardium. J. Am. Coll. Cardiol. 69 (2), 131-143. doi:10.1016/j.jacc. 2016.10.058

Hulsmans, M., Sager, H. B., Roh, J. D., Valero-Muñoz, M., Houstis, N. E., Iwamoto, Y., et al. (2018). Cardiac Macrophages Promote Diastolic Dysfunction. J. Exp. Med. 215 (2), 423-440. doi:10.1084/jem.20171274 
Igase, M., Kohara, K., Nagai, T., Miki, T., and Ferrario, C. M. (2008). Increased Expression of Angiotensin Converting Enzyme 2 in Conjunction with Reduction of Neointima by Angiotensin II Type 1 Receptor Blockade. Hypertens. Res. 31 (3), 553-559. doi:10.1291/hypres.31.553

Kanellakis, P., Ditiatkovski, M., Kostolias, G., and Bobik, A. (2012). A Pro-fibrotic Role for Interleukin-4 in Cardiac Pressure Overload. Cardiovasc. Res. 95 (1), 77-85. doi:10.1093/cvr/cvs142

Kaschina, E., Namsolleck, P., and Unger, T. (2017). AT2 Receptors in Cardiovascular and Renal Diseases. Pharmacol. Res. 125 (Pt A), 39-47. doi:10.1016/j.phrs.2017.07.008

Kikuchi, Y., Ikee, R., Hemmi, N., Hyodo, N., Saigusa, T., Namikoshi, T., et al. (2004). Fractalkine and its Receptor, CX3CR1, Upregulation in StreptozotocinInduced Diabetic Kidneys. Nephron Exp. Nephrol. 97 (1), e17-25. doi:10.1159/ 000077594

Kim, S. R., Lee, Y. H., Lee, S. G., Kang, E. S., Cha, B. S., Kim, J. H., et al. (2016). Urinary N-Acetyl- $\beta$-D-Glucosaminidase, an Early Marker of Diabetic Kidney Disease, Might Reflect Glucose Excursion in Patients with Type 2 Diabetes. Medicine (Baltimore) 95 (27), e4114. doi:10.1097/MD. 0000000000004114

Koenig, L. M., Boehmer, D. F. R., Metzger, P., Schnurr, M., Endres, S., and Rothenfusser, S. (2020). Blocking Inflammation on the Way: Rationale for CXCR2 Antagonists for the Treatment of COVID-19. J. Exp. Med. 217 (9), e20201342. doi:10.1084/jem.20201342

Krishnasamy, R., Isbel, N. M., Hawley, C. M., Pascoe, E. M., Burrage, M., Leano, R., et al. (2015). Left Ventricular Global Longitudinal Strain (GLS) Is a Superior Predictor of All-Cause and Cardiovascular Mortality when Compared to Ejection Fraction in Advanced Chronic Kidney Disease. PLoS One 10, e0127044. doi:10.1371/journal.pone.0127044

Kumar, V., Knowle, D., Gavini, N., and Pulakat, L. (2002). Identification of the Region of AT2 Receptor Needed for Inhibition of the AT1 Receptor-Mediated Inositol 1, 4, 5-triphosphate Generation. FEBS Lett. 532, 379-386. doi:10.1016/ s0014-5793(02)03713-4

Kurdi, A. T., Bassil, R., Olah, M., Wu, C., Xiao, S., Taga, M., et al. (2016). Tiam1/ Racl Complex Controls Il17a Transcription and Autoimmunity. Nat. Commun. 7, 13048. doi:10.1038/ncomms 13048

Kurisu, S., Ozono, R., Oshima, T., Kambe, M., Ishida, T., Sugino, H., et al. (2003). Cardiac Angiotensin II Type 2 Receptor Activates the Kinin/NO System and Inhibits Fibrosis. Hypertension 41, 99-107. doi:10.1161/01.hyp.0000050101. 90932.14

Lee, J. S., Yang, D. K., Park, J. H., Kim, J. O., Park, W. J., Cho, C., et al. (2017). MicroRNA-101b Attenuates Cardiomyocyte Hypertrophy by Inhibiting Protein Kinase C Epsilon Signaling. FEBS Lett. 591 (1), 16-27. doi:10.1002/ 1873-3468.12508

Leung, C. C. T., and Wong, C. K. C. (2021). Characterization of Stanniocalcin-1 Expression in Macrophage Differentiation. Transl Oncol. 14 (1), 100881. doi:10. 1016/j.tranon.2020.100881

Levick, S. P., and Widiapradja, A. (2020). The Diabetic Cardiac Fibroblast: Mechanisms Underlying Phenotype and Function. Int. J. Mol. Sci. 21 (3), 970. doi:10.3390/ijms 21030970

Li, R., Wang, H., Ma, F., Cui, G. L., Peng, L. Y., Li, C. Z., et al. (2021). Widespread Myocardial Dysfunction in COVID-19 Patients Detected by Myocardial Strain Imaging Using 2-D Speckle-Tracking Echocardiography. Acta Pharmacol. Sin. 1-8. doi:10.1038/s41401-020-00595-z

Li, Y., Hiroi, Y., and Liao, J. K. (2010). Notch Signaling as an Important Mediator of Cardiac Repair and Regeneration after Myocardial Infarction. Trends Cardiovasc. Med. 20 (7), 228-231. doi:10.1016/j.tcm.2011.11.006

Liu, J. H., Chen, Y., Yuen, M., Zhen, Z., Chan, C. W., Lam, K. S., et al. (2016). Incremental Prognostic Value of Global Longitudinal Strain in Patients with Type 2 Diabetes Mellitus. Cardiovasc. Diabetol. 15, 22. doi:10.1186/s12933-0160333-5

Liu, Q., Hou, A., Zhang, Y., Guo, Y., Li, J., Yao, Y., et al. (2019). MiR-190a Potentially Ameliorates Postoperative Cognitive Dysfunction by Regulating Tiam1. BMC Genomics 20 (1), 670. doi:10.1186/s12864-019-6035-0

Liu, Q., Qiao, W. H., Li, F. F., Deng, P., and Hu, Z. W. (2015). The Role of Interleukin-13 in Patients with Rheumatic Valvular Fibrosis: A Clinical and Histological Study. J. Heart Valve Dis. 24 (4), 496-501.

Luc, G., Arveiler, D., Evans, A., Amouyel, P., Ferrieres, J., Bard, J. M., et al. (2003). Circulating Soluble Adhesion Molecules ICAM-1 and VCAM-1 and Incident
Coronary Heart Disease: the PRIME Study. Atherosclerosis 170 (1), 169-176. doi:10.1016/s0021-9150(03)00280-6

Luck, C., DeMarco, V. G., Mahmood, A., Gavini, M. P., and Pulakat, L. (2017). Differential Regulation of Cardiac Function and Intracardiac Cytokines by Rapamycin in Healthy and Diabetic Rats. Oxid Med. Cel. Longev. 2017, 5724046. doi:10.1155/2017/5724046

Lum-Naihe, K., Toedebusch, R., Mahmood, A., Bajwa, J., Carmack, T., Kumar, S. A., et al. (2017). Cardiovascular Disease Progression in Female Zucker Diabetic Fatty Rats Occurs via Unique Mechanisms Compared to Males. Sci. Rep. 7, 17823. doi:10.1038/s41598-017-18003-8

Maack, C., Lehrke, M., Backs, J., Heinzel, F. R., Hulot, J. S., Marx, N., et al. (2018). Heart Failure and Diabetes: Metabolic Alterations and Therapeutic Interventions: a State-Of-The-Art Review from the Translational Research Committee of the Heart Failure AssociationEuropean Society of Cardiology. Eur. Heart J. 39 (48), 4243-4254. doi:10. 1093/eurheartj/ehy596

Mahmood, A., and Pulakat, L. (2015). Differential Effects of Beta-Blockers, Angiotensin II Receptor Blockers, and a Novel AT2R Agonist NP-6A4 on Stress Response of Nutrient-Starved Cardiovascular Cells. PLoS One 10, e0144824. doi:10.1371/journal.pone.0144824

Mangalmurti, N., and Hunter, C. A. (2020). Cytokine Storms: Understanding COVID-19. Immunity 53 (1), 19-25. doi:10.1016/j.immuni.2020.06.017

Martinelli, I., Tomassoni, D., Moruzzi, M., Roy, P., Cifani, C., Amenta, F., et al. (2020). Cardiovascular Changes Related to Metabolic Syndrome: Evidence in Obese Zucker Rats. Int. J. Mol. Sci. 21 (6), 2035. doi:10.3390/ijms21062035

Mauvais-Jarvis, F. (2020). Aging, Male Sex, Obesity, and Metabolic Inflammation Create the Perfect Storm for COVID-19. Diabetes 69 (9), 1857-1863. doi:10. 2337/dbi19-0023

Melenovsky, V., Andersen, M. J., Andress, K., Reddy, Y. N., and Borlaug, B. A. (2015). Lung Congestion in Chronic Heart Failure: Haemodynamic, Clinical, and Prognostic Implications. Eur. J. Heart Fail. 17 (11), 1161-1171. doi:10. 1002/ejhf.417

Nistala, R., Raja, A., and Pulakat, L. (2017). mTORC1 Inhibitors Rapamycin and Metformin Affect Cardiovascular Markers Differentially in ZDF Rats. Can. J. Physiol. Pharmacol. 95 (3), 281-287. doi:10.1139/cjpp-2016-0567

Niu, J., and Kolattukudy, P. E. (2009). Role of MCP-1 in Cardiovascular Disease: Molecular Mechanisms and Clinical Implications. Clin. Sci. (Lond) 117 (3), 95-109. doi:10.1042/CS20080581

Oktay, A. A., Aktürk, H. K., Paul, T. K., O’Keefe, J. H., Ventura, H. O., Koch, C. A., et al. (2000). "Diabetes, Cardiomyopathy, and Heart Failure," in Endotext [Internet]. Editors K. R. Feingold (South Dartmouth, MA: MDText.com, Inc.). PMID: 32776639.

Pan, Z., Sun, X., Shan, H., Wang, N., Wang, J., Ren, J., et al. (2012). MicroRNA-101 Inhibited Postinfarct Cardiac Fibrosis and Improved Left Ventricular Compliance via the FBJ Osteosarcoma Oncogene/transforming Growth Factor-B1 Pathway. Circulation 126 (7), 840-850. doi:10.1161/ CIRCULATIONAHA.112.094524

Pau, G., Fuchs, F., Sklyar, O., Boutros, M., and Huber, W. (2010). EBImage--an R Package for Image Processing with Applications to Cellular Phenotypes. Bioinformatics 26, 979-981. doi:10.1093/bioinformatics/btq046

Peiró, C., Lorenzo, Ó., Carraro, R., and Sánchez-Ferrer, C. F. (2017). IL-1 $\beta$ Inhibition in Cardiovascular Complications Associated to Diabetes Mellitus. Front. Pharmacol. 8, 363. doi:10.3389/fphar.2017.00363

Petrey, A. C., Qeadan, F., Middleton, E. A., Pinchuk, I. V., Campbell, R. A., and Beswick, E. J. (2021). Cytokine Release Syndrome in COVID-19: Innate Immune, Vascular, and Platelet Pathogenic Factors Differ in Severity of Disease and Sex. J. Leukoc. Biol. 109 (1), 55-66. doi:10.1002/JLB. 3COVA0820-410RRR

Piché, M. E., Tchernof, A., and Després, J. P. (2020). Obesity Phenotypes, Diabetes, and Cardiovascular Diseases. Circ. Res. 126 (11), 1477-1500. doi:10.1161/ CIRCRESAHA.120.316101

Qi, Y., Li, H., Shenoy, V., Li, Q., Wong, F., Zhang, L., et al. (2012). Moderate Cardiac-Selective Overexpression of Angiotensin II Type 2 Receptor Protects Cardiac Functions from Ischaemic Injury. Exp. Physiol. 97, 89-101. doi:10. 1113/expphysiol.2011.060673

Reifenberg, K., Lehr, H. A., Torzewski, M., Steige, G., Wiese, E., Küpper, I., et al. (2007). Interferon-gamma induces chronic active myocarditis and cardiomyopathy in transgenic mice. Am J Pathol 171 (2), 463-472. 
Riaz, H., Khan, M. S., Siddiqi, T. J., Usman, M. S., Shah, N., Goyal, A., et al. (2018). Association between Obesity and Cardiovascular Outcomes: A Systematic Review and Meta-Analysis of Mendelian Randomization Studies. JAMA Netw. Open 1 (7), e183788. doi:10.1001/jamanetworkopen.2018.3788

Rolski, F., and Błyszczuk, P. (2020). Complexity of TNF- $\alpha$ Signaling in Heart Disease. J. Clin. Med. 9 (10), 3267. doi:10.3390/jcm9103267

Salvador, A. M., Nevers, T., Velázquez, F., Aronovitz, M., Wang, B., Abadía Molina, A., et al. (2016). Intercellular Adhesion Molecule 1 Regulates Left Ventricular Leukocyte Infiltration, Cardiac Remodeling, and Function in Pressure Overload-Induced Heart Failure. J. Am. Heart Assoc. 5 (3), e003126. doi:10. 1161/JAHA.115.003126

Santhanam, A. V., d'Uscio, L. V., and Katusic, Z. S. (2010). Cardiovascular Effects of Erythropoietin an Update. Adv. Pharmacol. 60, 257-285. doi:10.1016/B9780-12-385061-4.00009-X

Schmidt, F. M., Weschenfelder, J., Sander, C., Minkwitz, J., Thormann, J., Chittka, T., et al. (2015). Inflammatory Cytokines in General and central Obesity and Modulating Effects of Physical Activity. PLoS One 10 (3), e0121971. doi:10. 1371/journal.pone.0121971

Schumacher, S. M., and Naga Prasad, S. V. (2018). Tumor Necrosis Factor- $\alpha$ in Heart Failure: an Updated Review. Curr. Cardiol. Rep. 20 (11), 117. doi:10.1007/ s11886-018-1067-7

Sharma, N., Belenchia, A. M., Toedebusch, R., Pulakat, L., and Hans, C. P. (2020). AT2R Agonist NP-6A4 Mitigates Aortic Stiffness and Proteolytic Activity in Mouse Model of Aneurysm. J. Cel. Mol. Med. 24 (13), 7393-7404. doi:10.1111/ jcmm.15342Epub ahead of print PMID: 32420690; PMCID: PMC7339180.

Sharp, A. S., Tapp, R. J., Thom, S. A., Francis, D. P., Hughes, A. D., Stanton, A. V., et al. (2010). ASCOT Investigators. Tissue Doppler E/E' Ratio Is a Powerful Predictor of Primary Cardiac Events in a Hypertensive Population: an ASCOT Substudy. Eur. Heart J. 31 (6), 747-752. doi:10.1093/eurheartj/ehp498

Souza, B. S. F., Silva, D. N., Carvalho, R. H., Sampaio, G. L. A., Paredes, B. D., Aragão França, L., et al. (2017). Association of Cardiac Galectin-3 Expression, Myocarditis, and Fibrosis in Chronic Chagas Disease Cardiomyopathy. Am. J. Pathol. 187 (5), 1134-1146. doi:10.1016/j.ajpath.2017.01.016

Strain, W. D., and Paldánius, P. M. (2018). Diabetes, Cardiovascular Disease and the Microcirculation. Cardiovasc. Diabetol. 17 (1), 57. doi:10.1186/s12933-018-0703-2

Sumners, C., de Kloet, A. D., Krause, E. G., Unger, T., and Steckelings, U. M. (2015). Angiotensin Type 2 Receptors: Blood Pressure Regulation and End Organ Damage. Curr. Opin. Pharmacol. 21, 115-121. doi:10.1016/j.coph.2015.01.004

Takawale, A., Zhang, P., Patel, V. B., Wang, X., Oudit, G., and Kassiri, Z. (2017). Tissue Inhibitor of Matrix Metalloproteinase-1 Promotes Myocardial Fibrosis by Mediating CD63-Integrin $\beta 1$ Interaction. Hypertension 69 (6), 1092-1103. doi:10.1161/HYPERTENSIONAHA.117.09045

Tartof, S. Y., Qian, L., Hong, V., Wei, R., Nadjafi, R. F., Fischer, H., et al. (2020). Obesity and Mortality Among Patients Diagnosed with COVID-19: Results from an Integrated Health Care Organization. Ann. Intern. Med. M20-M3742. doi:10.7326/M20-3742

Thavendiranathan, P., Verhaert, D., Kendra, K. L., and Raman, S. V. (2011). Fulminant Myocarditis Owing to High-Dose Interleukin-2 Therapy for Metastatic Melanoma. Br. J. Radiol. 84 (1001), e99-e102. doi:10.1259/bjr/13448473

Toedebusch, R., Belenchia, A., and Pulakat, L. (2018). Cell-specific Protective Signaling Induced by the Novel AT2R-Agonist NP-6A4 on Human Endothelial and Smooth Muscle Cells. Front. Pharmacol. 9, 928. doi:10.3389/fphar.2018.00928

Torzewski, M., Wenzel, P., Kleinert, H., Becker, C., El-Masri, J., Wiese, E., et al. (2012). Chronic Inflammatory Cardiomyopathy of Interferon $\gamma$-overexpressing Transgenic Mice Is Mediated by Tumor Necrosis Factor- $\alpha$. Am. J. Pathol. 180 (1), 73-81. doi:10.1016/j.jpath.2011.09.006

Tousoulis, D., Koumallos, N., Antoniades, C., Antonopoulos, A. S., Bakogiannis, C., Milliou, A., et al. (2010). Genetic Polymorphism on Type 2 Receptor of Angiotensin II, Modifies Cardiovascular Risk and Systemic Inflammation in Hypertensive Males. Am. J. Hypertens. 23 (3), 237-242. doi:10.1038/ajh.2009.233

Van Tassell, B. W., Trankle, C. R., Canada, J. M., Carbone, S., Buckley, L., Kadariya, D., et al. (2018). IL-1 Blockade in Patients with Heart Failure with Preserved Ejection Fraction. Circ. Heart Fail. 11 (8), e005036. doi:10.1161/ CIRCHEARTFAILURE. 118.005036

Virani, S. S., Alonso, A., Aparicio, H. J., Benjamin, E. J., Bittencourt, M. S., Callaway, C. W., et al. (2021). Heart Disease and Stroke Statistics-2021update: a Report from the American Heart Association. Circulation 143 (8), e254-e743. doi:10.1161/CIR.0000000000000950
Vogel, B., Siebert, H., Hofmann, U., and Frantz, S. (2015). Determination of Collagen Content within Picrosirius Red Stained Paraffin-Embedded Tissue Sections Using Fluorescence Microscopy. MethodsX 2, 124-134. doi:10.1016/j. mex.2015.02.007

Vora, A., de Lemos, J. A., Ayers, C., Grodin, J. L., and Lingvay, I. (2019). Association of Galectin-3 with Diabetes Mellitus in the Dallas Heart Study. J. Clin. Endocrinol. Metab. 104 (10), 4449-4458. doi:10.1210/jc.2019-00398

Wan, Y., Wallinder, C., Plouffe, B., Beaudry, H., Mahalingam, A. K., Wu, X., et al. (2004). Design, Synthesis, and Biological Evaluation of the First Selective Nonpeptide AT2 Receptor Agonist. J. Med. Chem. 47, 5995-6008. doi:10. 1021 /jm049715t

Wang, L., Zhang, Y. L., Lin, Q. Y., Liu, Y., Guan, X. M., Ma, X. L., et al. (2018). CXCL1-CXCR2 axis Mediates Angiotensin II-Induced Cardiac Hypertrophy and Remodelling through Regulation of Monocyte Infiltration. Eur. Heart J. 39 (20), 1818-1831. doi:10.1093/eurhearti/ehy085

Weil, B. R., and Neelamegham, S. (2019). Selectins and Immune Cells in Acute Myocardial Infarction and Post-infarction Ventricular Remodeling: Pathophysiology and Novel Treatments. Front. Immunol. 10, 300. doi:10. 3389/fimmu.2019.00300

Wickham, H. (2016). ggplot2: Elegant Graphics for Data Analysis. New York: Springer-Verlag. 978-3-319-24277-4. https://www.who.int/news/item/09-122020-who-reveals-leading-causes-of-death-and-disability-worldwide-2000-2019

Xuan, W., Liao, Y., Chen, B., Huang, Q., Xu, D., Liu, Y., et al. (2011). Detrimental Effect of Fractalkine on Myocardial Ischaemia and Heart Failure. Cardiovasc. Res. 92 (3), 385-393. doi:10.1093/cvr/cvr221

Yu, B., and Song, B. (2014). Notch 1 Signalling Inhibits Cardiomyocyte Apoptosis in Ischaemic Postconditioning. Heart Lung Circ. 23 (2), 152-158. doi:10.1016/j. hlc.2013.07.004

Zafiriou, M. P., Noack, C., Unsöld, B., Didie, M., Pavlova, E., Fischer, H. J. P., et al. (2014). Erythropoietin Responsive Cardiomyogenic Cells Contribute to Heart Repair post Myocardial Infarction. Stem Cells 32 (9), 2480-2491. doi:10.1002/stem.1741

Zagorski, J., Gellar, M. A., Obraztsova, M., Kline, J. A., and Watts, J. A. (2007). Inhibition of CINC-1 Decreases Right Ventricular Damage Caused by Experimental Pulmonary Embolism in Rats. J. Immunol. 179 (11), 7820-7826. doi:10.4049/jimmunol.179.11.7820

Zhang, Y., Wang, J. H., Zhang, Y. Y., Wang, Y. Z., Wang, J., Zhao, Y., et al. (2016). Deletion of Interleukin-6 Alleviated Interstitial Fibrosis in StreptozotocinInduced Diabetic Cardiomyopathy of Mice through Affecting TGF $\beta 1$ and miR-29 Pathways. Sci. Rep. 6, 23010. doi:10.1038/srep23010

Zhao, W., Zhao, T., Huang, V., Chen, Y., Ahokas, R. A., and Sun, Y. (2011). Platelet-derived Growth Factor Involvement in Myocardial Remodeling Following Infarction. J. Mol. Cel. Cardiol. 51 (5), 830-838. doi:10.1016/j. yjmcc.2011.06.023

Zhou, X., Ma, L., Habibi, J., Whaley-Connell, A., Hayden, M. R., Tilmon, R. D., et al. (2010). Nebivolol Improves Diastolic Dysfunction and Myocardial Remodeling through Reductions in Oxidative Stress in the Zucker Obese Rat. Hypertension 55 (4), 880-888. doi:10.1161/HYPERTENSIONAHA.109.145136

Zhu, R. R., Chen, Q., Liu, Z. B., Ruan, H. G., Wu, Q. C., and Zhou, X. L. (2020). Inhibition of the Notch1 Pathway Induces Peripartum Cardiomyopathy. J. Cel. Mol. Med. 24 (14), 7907-7914. doi:10.1111/jcmm.15423

Zoppini, G., Bergamini, C., Mantovani, A., Dauriz, M., Targher, G., Rossi, A., et al. (2018). The E/e' ratio difference between subjects with type 2 diabetes and controls. A meta-analysis of clinical studies. PLoS One 13 (12), e0209794. doi:10.1371/journal.pone.0209794

Conflict of Interest: Author MG was employed by the company Novopyxis Inc.

The remaining authors declare that the research was conducted in the absence of any commercial or financial relationships that could be construed as a potential conflict of interest.

Copyright (c) 2021 Gavini, Mahmood, Belenchia, Beauparlant, Kumar, Ardhanari, DeMarco and Pulakat. This is an open-access article distributed under the terms of the Creative Commons Attribution License (CC BY). The use, distribution or reproduction in other forums is permitted, provided the original author $(s)$ and the copyright owner(s) are credited and that the original publication in this journal is cited, in accordance with accepted academic practice. No use, distribution or reproduction is permitted which does not comply with these terms. 